\title{
Diabatic generation of negative potential vorticity and its impact on the North Atlantic jet stream
}

Article

Accepted Version

Harvey, B., Methven, J., Sanchez, C. and Schaefler, A. (2020) Diabatic generation of negative potential vorticity and its impact on the North Atlantic jet stream. Quarterly Journal of the Royal Meteorological Society, 146 (728). pp. 1477-1497. ISSN 1477-870X doi: https://doi.org/10.1002/qj.3747 Available at https://centaur.reading.ac.uk/89119/

It is advisable to refer to the publisher's version if you intend to cite from the work. See Guidance on citing.

To link to this article DOI: http://dx.doi.org/10.1002/qj.3747

Publisher: Royal Meteorological Society

All outputs in CentAUR are protected by Intellectual Property Rights law, including copyright law. Copyright and IPR is retained by the creators or other copyright holders. Terms and conditions for use of this material are defined in the End User Agreement.

www.reading.ac.uk/centaur 
Central Archive at the University of Reading

Reading's research outputs online 


\title{
Diabatic generation of negative potential vorticity and its impact on the North Atlantic jet stream
}

\author{
Ben Harvey ${ }^{1,2}$ | John Methven ${ }^{2}$ | Claudio Sanchez ${ }^{3}$ | \\ Andreas Schäfler ${ }^{4}$
}

${ }^{1}$ National Centre for Atmospheric Science, UK

${ }^{2}$ Department of Meteorology, University of Reading, UK

${ }^{3}$ Met Office, UK

${ }^{4}$ Deutsches Zentrum für Luft- und Raumfahrt, Institut für Physik der Atmosphäre, Germany

Correspondence

Ben Harvey, Department of Meteorology, University of Reading, RG6 6BB, UK

Email: b.j.harvey@ncas.ac.uk

Funding information

$\mathrm{BH}$ was funded by the National Centre for Atmospheric Science

Localised regions of negative potential vorticity ${ }^{a}(\mathrm{PV})$ are frequently seen on the equatorward flank of the upper-tropospheric jet streams in analysis and forecast products. Their positioning, on the anticyclonic side of the jet and often close to the jet core, suggest they are associated with an enhancement of jet stream maximum winds. Given that PV is generally positive in the northern hemisphere and is conserved under adiabatic conditions, the presence of negative PV is indicative of recent diabatic activity. However, little is understood on the mechanisms for its generation and subsequent lifecycle. In this paper, aircraft measurements from a recent field campaign are used to provide direct observational evidence for the presence of negative PV on the anticyclonic side of an upper-tropospheric jet. Theory is then developed to understand the process by which PV can turn negative. The key ingredient is diabatic heating in the presence of vertical wind shear, and the resulting PV anomalies are shown to always result from a flux of PV directed 'down the isentropic slope'. This explains why, for the typical situation of heating in a warm conveyor belt, negative PV values appear on the equatorward side of the upper-tropospheric jet stream close to the jet core. These ideas are illustrated with a semi-geostrophic model and the processes responsible for the observed negative PV are explored using an 
operational forecast model with online PV tracer diagnostics. The diabatic influence on jet stream winds and shear is of interest because it is pertinent to the predictability of extreme jet stream events and associated flight-level turbulence, and is crucial to the propagation of Rossby waves at tropopause level, development of mid-latitude weather systems and their subsequent impacts at the surface.

\section{KEYWORDS}

Negative potential vorticity; diabatic heating; jet stream; jet streaks; impermeability theorem; symmetric instability

${ }^{a}$ The phrase 'negative PV' is used throughout from a northern hemisphere perspective; this is shorthand for negative $f P$, where $f$ is the Coriolis parameter and $P$ the PV.

\section{1 | INTRODUCTION}

Westerly jet streams are a major feature of the extratropical global circulation. In the zonal mean, there are times when only one jet maximum is identifiable in the Northern Hemisphere, but also times when the zonal mean jet is split into a subtropical jet and higher latitude jet (Eichelberger and Hartmann, 2007). The subtropical jet is highly baroclinic with only a weak surface westerly signature, while the higher latitude jet is more transient in nature and typically has a stronger barotropic component and surface westerlies. Due to the role of baroclinic wave momentum fluxes in reinforcing the high-latitude jet it has been called the "eddy-driven jet" in recent literature (e.g. Woollings et al., 2010). In the climatological time-average of winds at tropopause level, there is a distinct spiral of maximum winds extending from the subtropical Atlantic, across North Africa, Eurasia, the Pacific and North America returning across the North Atlantic in mid-latitudes (50-55 N, e.g. Hoinka, 1999). Therefore, there are two distinct jet streams in the longitude sector of the eastern North Atlantic. However, the time-average and zonal-average pictures hide the fact that there are typically very large-amplitude meridional excursions of the jet stream - a signature of Rossby wave activity at the tropopause. Locally the jet stream can be much stronger than the average, sometimes exceeding $100 \mathrm{~m} \mathrm{~s}^{-1}$ in the jet core, compared with $40 \mathrm{~m} \mathrm{~s}^{-1}$ maximum in the zonal-average. Such locally intense wind speeds, or "jet streaks", occur where the jet is relatively straight, most often on the western or northern flank of ridges in the meandering jet pattern (Pyle et al., 2004).

Jet stream cores are co-located with strong gradients in potential vorticity (PV) on a range of isentropic surfaces intersecting the tropopause. The subtropical jet occurs on higher isentropic surfaces than the eddy-driven jet consistent with the tropopause sloping upwards towards the edge of the tropics (e.g., see Hoskins and James (2014)). Therefore in a map of PV on one isentropic surface it is typical to see only one jet stream, even if it is highly contorted. A more compact depiction in a situation with multiple jets is obtained from maps of potential temperature $(\theta)$ on the "PV2 surface" where PV = 2 PVU (1 PVU = 10-6 $\mathrm{K} \mathrm{m}^{2} /(\mathrm{kg} \mathrm{s})$ ) (Hoskins and Berrisford, 1988; Nielsen-Gammon, 2001). The rate of propagation of Rossby waves, their dispersion (or group velocity) and the strength of interaction with lower levels all depend on the magnitude of the PV gradient across the tropopause (or equivalently $\theta$-gradient along the tropopause) and the wind speed at that location (Harvey et al., 2016).

Diabatic heating in warm conveyor belts (WCBs) is known to influence the jet stream via advection of the tropopause 
by the upper tropospheric divergence where WCBs outflow into jet stream ridges (Grams et al., 2011; Grams and Archambault, 2016). This process is an important contributor to the development of weather systems, and one suspected of suffering from inaccuracies in numerical weather prediction models (Martínez-Alvarado et al., 2014; Joos and Forbes, 2016), in particular relating to the vertical profile of latent heating associated with condensation of liquid water, parametrization of ice microphysics and interaction with radiative transfer. Rodwell et al. (2013) used a lagged composite of the 100 worst forecasts for Central Europe to show that there was a common precursor Rossby wave pattern 5-6 days before each "forecast bust". This pre-cursor pattern is characterised by a short-wave trough over the Rockies and poleward extension of warm, moist air from the Caribbean over the eastern side of the USA. They argued that misrepresentation of active mesoscale weather systems within the warm air mass results in forecast error in the tropopause ridge above and that this error propagates and grows downstream as a modification to the Rossby wave pattern, resulting in a major error over Europe.

There is also reason to suspect systematic misrepresentation of Rossby wave evolution in forecast models. Gray et al. (2014) have shown that the isentropic gradient of PV around the rims of ridges declines strongly with lead time during the first 1-2 days of forecasts in several leading operational NWP forecasts. Harvey et al. (2016) have shown analytically that Rossby waves must to propagate too slowly towards the east in such a situation. Furthermore, Gray et al. (2014) showed that the average amplitude of ridges also declines with lead time over the first 5 days of forecasts. This can be explained in terms of a nonlinear process involving excessive filamentation of PV on the flanks of the jet: if the transition of $\mathrm{PV}$ across the tropopause is too smooth then there is an enhanced meridional dispersion of wave activity away from the jet core (Harvey et al., 2018). Saffin et al. (2017) has shown using a PV tracer technique that there is a competition between the maintenance of $\mathrm{PV}$ contrast across the tropopause by parametrized physical processes and a reduction associated with non-conservation of PV by the dynamical core of the UK Met Office NWP model (MetUM). So forecast error in the tropopause gradient could arise through either the physical parametrizations or the dynamical core.

It is a major challenge to deduce from observations the influence of diabatic processes on the jet stream. Instruments measure state variables such as pressure, temperature and wind, rather than processes, such as diabatic heating. Typically a numerical model is used to deduce processes acting, given the data on the state. In data assimilation the new observations are blended with a previous forecast to create the analysis - an optimal estimate of the current atmospheric state. Then processes are deduced from running the model in forecast mode from initial conditions given by the analysis. However, how then do we deduce when the model misrepresents processes? One approach is to use theoretical considerations to rule out the influence of some processes on the evolution. In this paper, we will use material conservation of $\mathrm{PV}$ to separate adiabatic from diabatic processes and further to partition distinct mechanisms associated with the influence of diabatic processes on jet stream structure including maximum wind speed and shear.

On synoptic scales the net effect of positive diabatic heating rates is often described as a "dilution" of PV substance in the isentropic layers above the heating maximum, and a "concentration" below. This terminology is used because there can be no flux of PV across isentropic surfaces, but there can be a flux of mass (Haynes and McIntyre, 1990). To the extent that PV can be regarded as analogous to the concentration of an artificial chemical species, the effect of diabatic mass transport convergence (divergence) is to dilute (concentrate) that species. This puts a constraint on the generation of negative PV values on synoptic scales since the dilution process can reduce PV towards zero, but not change its sign. On smaller scales, localised regions of negative PV can be generated and their presence is consistent with studies on the mesoscale and smaller whereby heating anomalies in an environment with strong vertical wind shear are known to create quasi-horizontal PV dipoles, with no constraint on its sign (Chagnon and Gray, 2009; Oertel et al., in review). The quasi-horizontal dipoles arise from a second PV tendency term given by a non-advective PV flux along isentropic surfaces (Haynes and McIntyre, 1990). Slantwise WCB ascent lies at the boundary between the synoptic and 
mesoscales, and is typically positioned on the western side of ridges near the vertical wind shear of the jet stream. This paper aims to better understand when and how WCB heating can generate negative PV features at upper-tropospheric levels.

We first present direct observations of negative PV in the upper-troposphere from the recent North Atlantic Waveguide and Downstream Impacts Experiment aircraft field campaign (NAWDEX; Schäfler et al., 2018). A curtain of dropsondes across a strong linear jet streak is used to compute PV values, and in situ wind observations are used to verify the negative values (Sec. 3). We then proceed to explore the theory regarding the diabatic modification of PV in order to quantify the roles of the two terms in the generation of negative PV values (Sec. 4). The aim is to clarify the extent to which PV is constrained to remain positive on scales relevant for WCB outflow, and to understand when and how negative values are produced. The theoretical developments are next illustrated using simulations of an idealised frontal-geostrophic model with diabatic heating (Sec. 5), which is the simplest model containing the essential ingredients required to illustrate the theory. Finally, the processes responsible for the generation of the observed negative PV in Sec. 3 are identified through the use of detailed online PV tracer diagnostics in the MetUM (Sec. 6). Further discussion and the main conclusions are presented in Sec. 7.

\section{2 | MODEL DETAILS}

Two numerical models are used in this study to help link the observations to the theory: the operational version of the UK Met Office NWP model (MetUM) and the simplified frontal-geostrophic model with heating. These are described here for later reference.

\section{1 | The MetUM model and diabatic tracer diagnostics}

Hindcast simulations have been performed using the configuration of the MetUM which was operational at the time of the NAWDEX campaign, Global Atmosphere v6.1 (GA6.1, Walters et al., 2017). MetUM GA6.1 is a finite-difference model, using a semi-implicit, semi-Lagrangian integration scheme to solve the non-hydrostatic, deep-atmosphere equations. The dynamical core is the Even Newer Dynamics for General atmospheric modelling of the environment (ENDGame, Wood et al., 2014). The horizontal resolution employed is N768 (approx. $17 \mathrm{~km}$ grid spacing in the midlatitudes) with 70 levels in the vertical (approx. $500 \mathrm{~m}$ grid spacing at tropopause level). Parametrised physical processes include radiation (Edwards and Slingo, 1996), large-scale rain (Wilson and Ballard, 1999), turbulent mixing (Lock et al., 2000) and convection (Gregory and Rowntree, 1990).

The MetUM includes the diabatic tracer diagnostics developed by Gray (2006), Chagnon et al. (2013) and Saffin et al. (2016). These consist of a number of online tracer fields for both potential temperature and PV. For each variable there is one tracer $\varphi_{\text {adv }}$ (the 'advection-only' tracer) which is set to the initial field at the start of a simulation and then simply advected passively by the semi-Lagrangian transport scheme of the MetUM. The remaining tracers are all set to zero at the start of the simulation but each accumulate the local tendency of either potential temperature or PV due to one of the parametrised physical processes. As such, there is one potential temperature tracer and one PV tracer for each parametrised process.

The utility of the diabatic tracer diagnostics lies in the fact that at any time the full field $\varphi$ of either potential temperature or $\mathrm{PV}$ can be decomposed as

$$
\varphi(\mathbf{x}, t)=\varphi_{\mathrm{adv}}(\mathbf{x}, t)+\sum_{\mathrm{n}} \varphi_{\mathrm{n}}(\mathbf{x}, t)+\epsilon
$$


where the right hand side represents the sum of the advection-only tracer and the accumulated contribution from each of the physical processes (labelled $n$ ). In practice this decomposition is not exact, and the error is denoted $\epsilon$. For potential temperature, the error arises because of inaccuracies in the tracer scheme meaning the sum of several tracer variables does not necessarily remain equal to the tracer advection of their sum (the so-called 'splitting error' of Saffin et al., 2016). For PV the residual is more complex because, in addition to the splitting error, an error arises because the dynamical core does not exactly conserve PV. At each timestep numerical inaccuracies in the model's time-stepping scheme result in small modifications to the full PV field which are not accounted for by the other tracers. This issue has been explored in detail by Saffin et al. (2016) who implemented an additional PV tracer $\varphi_{i}(\mathrm{x}, t)$ (the 'dynamics-tracer inconsistency' tracer) which is also set to zero at the start of each simulation but accumulates an estimate of the error in PV introduced by the dynamical core at each timestep. As such for PV the decomposition can be taken further by writing

$$
\epsilon=\varphi_{i}(\mathrm{x}, t)+\text { residual }
$$

where the final residual term is shown to be approximately an order of magnitude smaller than $\varphi_{i}$ and $\varphi_{n}$ in Saffin et al. (2016). In the results that follow, the dynamics-tracer inconsistency is grouped with two additional PV tracers associated with the elliptic solver at the end of the timestep in the MetUM model, namely the pressure solver routine and the mass balance routine, although these terms are small in the case considered here. The sum of these three PV tracers is called the 'PV change due to the dynamical core'.

\section{2 | The frontal-geostrophic model}

The frontal-geostrophic model is the $2 \mathrm{D}$ version of the semi-geostrophic equations studied by Hoskins (1975). The 2D case was originally called semi-geostrophic by Hoskins (1971), however this term is now generally reserved for the full 3D system. Due to the symmetry of the 2D setup the frontal-geostrophic model is remarkably accurate, and it is the simplest model containing the essential ingredients required to illustrate the generation of negative PV by diabatic heating. A full derivation is provided in App. A; here a brief overview is provided to introduce notation.

The frontal-geostrophic system represents the evolution of a zonally-symmetric anelastic flow on an $f$-plane in which the zonal momentum $m=u-f y$ and potential temperature $\theta$ fields are advected in the meridional $(y, z)$ plane and optionally modified by heating $Q$ and friction $F$ :

$$
\begin{aligned}
& \frac{D m}{D t}=F \\
& \frac{D \theta}{D t}=Q
\end{aligned}
$$

where

$$
\frac{D}{D t}=\frac{\partial}{\partial t}+v \frac{\partial}{\partial y}+w \frac{\partial}{\partial z}
$$

is the Lagrangian derivative. Zonal advection drops out due to symmetry of the flow in the $x$-direction. The meridional circulation $(v, w)$ is obtained as the unique flow which maintains thermal wind balance between the zonal momentum and potential temperature fields at each time step, as described in App. A. The result is a balanced system governed by a 
single prognostic equation for the PV field:

$$
\rho P=m_{z} \theta_{y}-m_{y} \theta_{z}
$$

Note that, unlike in the quasi-geostrophic system, the full Ertel PV field is the relevant quantity here and it is advected by the full wind rather than the geostrophic wind. All other fields can be obtained from $P$ through inversion of the balance relationships, provided $f P$ remains positive (see App. A). Once $f P$ becomes negative somewhere in the domain the PV inversion ceases to be elliptic and the simulations stop.

\section{3 | OBSERVATIONS OF NEGATIVE PV AT JET STREAM LEVEL}

The North Atlantic Wave-guide and Downstream Impacts Experiment (NAWDEX) took place during autumn 2016. Multiaircraft and ground-based observations were made over the North Atlantic with the aim of better understanding the importance of diabatic processes for mid latitude weather (see Schäfler et al., 2018, for an overview of the observational experiment). The airborne payload contained numerous in situ instruments together with dropsondes and a range of remote sensing equipment including passive microwave radiometers, a water vapour differential absorption lidar, and Doppler wind lidars. The calculation of PV requires temperature and horizontal wind data with sufficient spatial resolution to allow accurate computation of both vertical and horizontal gradients. For this purpose the dropsondes are utilised because all variable measurements are co-located, although close dropsonde spacing is required to compute accurate horizontal gradients.

Several early studies estimated PV at jet stream level based entirely on radiosonde profiles (e.g. Danielsen and Mohnen, 1977; Danielsen et al., 1987) or using a combination of radiosonde profiles and aircraft observations (Shapiro, 1974, 1976; Shapiro et al., 1987). These observations did not, however, have sufficient resolution for the accurate diagnosis of negative PV features. In this section, a curtain of 22 dropsondes with an average spacing of $29 \mathrm{~km}$ on one straight flight leg across the jet stream is used to compute a PV cross section. This provides direct evidence for the presence of negative PV values in the upper-troposphere, manifested by an anticyclonic shear so strong that the vertical component of absolute vorticity is negative. The robustness of this result is then explored using high-resolution in situ wind measurements along the flight track.

\section{1 | Outline of synoptic situation and aircraft observations}

The case considered is the post-tropical stage of tropical storm Karl. Karl was a long-lived tropical system which reached tropical storm intensity on 15 September 2016 and remained in the western tropical Atlantic for 10 days (Pasch and Zelinsky, 2016; Euler et al., 2019). It then progressed northwards and interacted with the jet stream on 26 September (Fig. 1a). It moved under a pre-existing jet streak before transitioning rapidly into an extratropical cyclone. The jet streak was enhanced during the extra-tropical transition, with a wind speed maximum of $90 \mathrm{~m} \mathrm{~s}^{-1}$ extending to the north of Scotland on 27 September (Fig. 1c). The associated poleward moisture transport and strong surface winds resulted in widespread flooding in Norway on 28 and 29 September (Schäfler et al., 2018).

Three NAWDEX flights took place on 27 September: The HALO and DLR Falcon aircraft flew from Keflavik Airport, Iceland, and the FAAM BAe146 flew from East Midlands Airport, UK. All three aircraft made transects of the jet streak, and FAAM and DLR Falcon aircraft performed a coordinated leg to the west of Scotland just before midday (Figs. 1c, d). Together with a HALO flight further west on the previous day, and independent flights made earlier in the week by the 
TAB LE 1 Details of the flight track sections referred to in the text.

\begin{tabular}{|ccccccc|} 
Point Label & Aircraft & Time [UTC] & Longitude & Latitude & Altitude & Air Pressure \\
\hline A & FAAM & $10: 43$ & $7.5 \mathrm{~W}$ & $62.5 \mathrm{~N}$ & $8.38 \mathrm{~km}$ & $330 \mathrm{hPa}$ \\
B & FAAM & $11: 53$ & $7.7 \mathrm{~W}$ & $57.0 \mathrm{~N}$ & $9.68 \mathrm{~km}$ & $289 \mathrm{hPa}$ \\
\hline C & HALO & $12: 21$ & $29.0 \mathrm{~W}$ & $60.4 \mathrm{~N}$ & $8.45 \mathrm{~km}$ & $330 \mathrm{hPa}$ \\
D & HALO & $15: 17$ & $29.0 \mathrm{~W}$ & $45.4 \mathrm{~N}$ & $9.04 \mathrm{~km}$ & $330 \mathrm{hPa}$ \\
\hline
\end{tabular}

unmanned NASA Global Hawk aircraft (as part of the NOAA SHOUT campaign) before the extra-tropical transition occurred, these observations provide a unique dataset covering the full lifecycle of the extratropical transition of a cyclone with re-intensification (Schäfler et al., 2018).

The focus in this section is the FAAM flight which traversed the jet streak just before midday on 27 September (flight number B981). The transect starts at point A in Fig. $1 \mathrm{~d}$ at pressure level $330 \mathrm{hPa}$ ( $8.38 \mathrm{~km}$ altitude) and heads south towards point $\mathrm{B}$, ascending to pressure level $290 \mathrm{hPa}$ ( $9.68 \mathrm{~km}$ altitude) part way along. Further details of the flight track are presented in Tab. 1. The aircraft measured in situ wind, temperature, pressure, humidity, and ozone, and released 22 dropsondes at an average interval of $29 \mathrm{~km}$ across the transect. The wind, potential temperature and humidity data from the dropsondes are plotted in Fig. 2 . The dropsonde measurements are reported every $0.5 \mathrm{~s}$ giving a vertical resolution of 5-10 m. To produce these sections, the dropsonde profiles are first smoothed in the vertical using a Gaussian kernel smoother with half-width of $250 \mathrm{~m}$ and then re-gridded onto a regular $50 \mathrm{~m}$ vertical grid before being linearly interpolated in the horizontal direction to provide data on a uniform 2D grid for plotting. The vertical smoothing has relatively little impact on the fields shown in Fig. 2, but is required to reduce noise associated with the high vertical resolution, which becomes problematic when vertical derivatives are taken (see next section). This choice of smoothing is motivated by noting that large-scale atmospheric features typically have aspect ratios scaling as $f / N \approx 0.01$. Since the sonde spacing is around $25 \mathrm{~km}$ in the horizontal, a vertical smoothing of $250 \mathrm{~m}$ results in a similar effective aspect ratio as the observational features. However, a range of different smoothing half-widths have been tested and the detection of negative PV and other general conclusions do not depend sensitively on this choice, as shown below.

Key points to note from Fig. 2 are: the zonally-oriented jet streak between $58 \mathrm{~N}$ and $59 \mathrm{~N}$ with a strong baroclinic zone beneath; the presence of very strong cyclonic shear $(\approx 3 f)$ to the north of the jet maximum and weaker anticyclonic shear $(\approx-1 f)$ to the south; a second region of strong cyclonic shear between $61 \mathrm{~N}$ and $62 \mathrm{~N}$ that is more barotropic in nature. Finally, the relative humidity field (Fig. 2c) hints at the tropopause structure: a southward-sloping intrusion of dry stratospheric air lies beneath the jet maximum and a second more vertically-oriented dry-air intrusion is present at $61-62 \mathrm{~N}$.

\section{2 | PV computed from the dropsondes}

PV cannot be measured by point observations at a single location because it requires the calculation of spatial derivatives of wind and potential temperature. Knowledge of $3 \mathrm{D}$ fields is usually required to compute $\mathrm{PV}$ accurately. In the present case, however, there is very little variation in the zonal direction (see Figs. 1c, d), so the 2D form of (6) is used, written as

$$
\rho P=\left(f-u_{y}\right) \theta_{z}+\left(f_{\text {hor }}+u_{z}\right) \theta_{y}
$$


where $\rho$ is air density, $f=2 \Omega \sin$ (latitude) is the Coriolis parameter, $u$ the wind speed in the $x$-direction, $\theta$ the potential temperature, $y$ the cross-jet distance and $z$ altitude. The variable $f_{\text {hor }}=2 \Omega \cos$ (latitude) is the horizontal component of planetary vorticity which in general contributes very little to the full PV field, but is included here for completeness. All other terms in the Ertel PV field (e.g. those involving $\theta_{x}, v_{x}$ and $w_{x}$ ) vanish due to the assumed zonal symmetry.

Equation 7 is evaluated from the kernel-smoothed sonde profiles as follows. The horizontal gradients $u_{y}$ and $\theta_{y}$ are evaluated at the midpoint between each consecutive pair of sondes using a centred difference. The vertical gradients $u_{z}$ and $\theta_{z}$ are evaluated along each sonde profile in turn before being interpolated horizontally onto the same midpoint locations. The PV is then computed on this 'PV grid' before being linearly interpolated to a full $2 \mathrm{D}$ grid for plotting. The result is shown in Fig. 3. As hinted by the relative humidity field in Fig. 2c, the PV field shows two tropopause folds: one sloping southward beneath the jet maximum and a more vertically-aligned structure at $61-62 \mathrm{~N}$. In both cases the dynamical tropopause, as measured by the $2 \mathrm{PVU}$ surface, reaches down to around $5 \mathrm{~km}$.

Of interest for the present study are the two regions with negative PV values: one just south of the jet maximum at $57.5 \mathrm{~N}$ and 8-9 $\mathrm{km}$ altitude, and one just north of the barotropic fold at $62 \mathrm{~N}$ and $4-6 \mathrm{~km}$ altitude. In both cases the PV values are only just below zero, however the change of its sign is robust, as demonstrated in Sec. 3.3. To understand better the nature of the negative PV, Figs. $3 \mathrm{~b}$ and $3 \mathrm{c}$ show the full PV field decomposed as the two terms on the right hand side of (7). These terms represent the PV associated with the vertical and horizontal components of absolute vorticity respectively. As might be expected, the horizontal vorticity term (final term in (7); Fig. 3c) is everywhere negative, or near zero. This is because, to the extent that thermal wind balance holds, $u_{z} \propto-\theta_{y}$. Therefore this term can be expected to always be negative and only large along the strong baroclinic zone beneath the jet maximum. However,this term is not responsible for either of the two regions with negative $\mathrm{PV}$ values because it is near zero in those regions. The vertical component of absolute vorticity (first term on the right hand side of (7)), in contrast, clearly exhibits negative PV structures of the same shape and magnitude as the full PV field (Fig. 3b).

\section{3 | Discussion of the robustness of the negative PV values}

The observations presented above provide evidence of negative PV in the upper-troposphere on the anti-cyclonic flank of the jet stream. However, the values are only just below zero. In this section the robustness of the observed negative PV values are explored, first by testing their sensitivity to the amount of vertical smoothing applied to the dropsonde data, and then by analysing high-frequency in situ wind observations from the aircraft for further evidence that the vertical component of absolute vorticity is indeed negative in those regions.

The PV section in Fig. 3a was obtained from the drop sonde data after applying a Gaussian kernel smoothing in the vertical direction with half-width of $250 \mathrm{~m}$. This computation has been repeated for a range of smoothing half-widths, and the results are presented in the Supplementary Material. Figure S1 shows that, other than the amount of noise present, the smoothing does not modify the structure of the PV field much and, crucially, the presence of the two regions of negative PV is clearly present in each case and independent of the amount of smoothing applied.

As an independent verification that the PV is negative, Fig. 4 shows high-frequency $(1 \mathrm{~Hz})$ in situ wind and ozone observations from the FAAM aircraft. Whilst PV cannot be computed from observations along a single 1D flight track, the fact that the vertical component of absolute vorticity turns negative can be verified. As above, the in situ wind data are first smoothed with a Gaussian kernel filter to allow cleaner finite differences to be computed. Figure 4a shows the in situ wind speeds (red) together with wind speeds from the dropsonde section in Fig. 2a at heights $8.5 \mathrm{~km}$ (blue) and $5 \mathrm{~km}$ (green). The in situ values are generally similar to the $8.5 \mathrm{~km}$ section south of $60.5 \mathrm{~N}$ and similar to the $5 \mathrm{~km}$ section north of $61 \mathrm{~N}$, despite the altitude difference between the aircraft and the sonde measurements. The in situ winds however exhibit a striking stepped structure on the north side of the jet with a spatial scale of around $30 \mathrm{~km}$ 
which is not resolved by the dropsonde data.

Figure $4 \mathrm{~b}$ shows the corresponding values of the vertical component of absolute vorticity, $f-u_{y}$. Both the in situ wind data and the relevant section from the sondes show that the vertical component of absolute vorticity turns negative in the two regions of negative PV. The fine-scale structure noted in the in situ wind data of Fig. 4a is also clearly evident in Fig. 4b as bands of high vorticity, of too small a scale to be captured by the dropsondes. Fig. 4c shows that the ozone concentration along the flight leg also exhibits similar variations, with bands of high ozone coincident with the bands of high vorticity. These bands are marked by the vertical lines (i)-(iv) in all panels. Together these observations suggest a layering of filaments of stratospheric air parallel to the tropopause near the jet stream core (as observed by Vaughan et al., 2001). Marker (iv) is on the stratospheric side of the PV gradient zone, and marker (i) is on the tropospheric side and located near the jet core. The full tropopause transition region in terms of ozone is $\approx 100 \mathrm{~km}$ across. The largest PV gradient, however, is confined to the region of width $\approx 30 \mathrm{~km}$ between markers (i) and (ii).

\section{I HOW CAN NEGATIVE PV ARISE IN THE FLOW?}

Given the evidence presented in the previous section for the presence of negative PV in observations, the aim of this section is to understand the mechanism by which negative PV is produced. This is achieved using the isentropic form the PV evolution equation which is derived in Sec. 4.1 and examined in Sec. 4.2.

\section{1 | PV dilution and the non-advective PV flux}

The evolution of PV in the presence of diabatic heating and friction is compactly written in Lagrangian form as:

$$
\begin{aligned}
\rho \frac{D P}{D t} & =\boldsymbol{\zeta} \cdot \nabla Q-\nabla \times \mathbf{F} \cdot \nabla \theta \\
& =\nabla \cdot(\boldsymbol{\zeta} Q-\mathbf{F} \times \nabla \theta)
\end{aligned}
$$

where the Lagrangian derivative following the full 3-D wind field, $\mathbf{u}$, is:

$$
\frac{D}{D t}=\frac{\partial}{\partial t}+\mathbf{u} \cdot \nabla
$$

$P=\zeta \cdot \nabla \theta / \rho$ is the Ertel PV, $\zeta$ is the absolute vorticity vector, $Q$ is the Lagrangian rate of change of potential temperature due to diabatic heating and $\mathbf{F}$ represents frictional forces.

Equation 8 shows that PV is conserved along flow trajectories in the absence of diabatic heating and friction. When diabatic heating is present the instantaneous Lagrangian PV tendency can be viewed as a flux directed along absolute vortex lines (e.g. Raymond, 1992). As such, this full Lagrangian form of the PV equation provides a simple extension to the familiar concept of Lagrangian conservation of PV from adiabatic dynamics, and it has been utilised extensively by a large number of studies examining the influence of diabatic heating on the dynamics of weather systems (e.g. Madonna et al., 2014; Joos and Forbes, 2016; Martínez-Alvarado et al., 2014). However, in this form there are no obvious constraints to the change in value or sign of PV along a trajectory. Moreover, as first noted by Haynes and McIntyre (1987), whenever diabatic heating causes an air parcel to traverse an isentropic surface there is an exact cancellation between the cross-isentropic advection of PV by the air parcel and the diabatic modification of PV in the surrounding isentropic layers: the mass-weighted integral of PV within each isentropic layer must remain exactly unchanged. 
The proof, following Haynes and McIntyre (1987), proceeds by considering a flux form of the PV equation. First define $\mathbf{n}=\nabla \theta /|\nabla \theta|$ as the unit vector normal to isentropic surfaces, and $\mathbf{u}_{D}=\mathbf{n} Q /|\nabla \theta|$ as the diabatic velocity component such that $\rho \mathbf{u}_{D}$ is the diabatic mass flux across isentropic surfaces. Next split the absolute vorticity vector into components pointing across and along the local isentropic surface: $\boldsymbol{\zeta}=(\boldsymbol{\zeta} \cdot \mathbf{n}) \mathbf{n}+\boldsymbol{\zeta}_{/ /}$, where $\boldsymbol{\zeta}_{/ /}=\mathbf{n} \times(\boldsymbol{\zeta} \times \mathbf{n})$ is the projection of $\zeta$ onto the local isentropic surface. The term $\boldsymbol{\zeta} \cdot \mathbf{n}$ is proportional to the $\mathrm{PV}$ and multiplying by $Q$ gives the identity:

$$
\zeta Q=\rho P \mathbf{u}_{D}+\zeta_{/ /} Q
$$

Combining (8), (9) and (10), the PV equation can be re-written in the flux form:

$$
\frac{\partial(\rho P)}{\partial t}+\nabla \cdot\left(\rho P \mathbf{V}-\boldsymbol{\zeta}_{/ /} Q+\mathbf{F} \times \nabla \theta\right)=0
$$

where $\mathbf{V}=\mathbf{u}-\mathbf{u}_{D}$ is the component of velocity parallel to isentropic surfaces. The expression within the divergence is the complete, unapproximated PV flux and each of the three terms in the flux is parallel to isentropic surfaces by definition. Therefore,

$$
\left(\rho P \mathbf{V}-\boldsymbol{\zeta}_{/ /} Q+\mathbf{F} \times \nabla \theta\right) \cdot \mathbf{n}=0
$$

This remarkable result was described by Haynes and Mclntyre (1990) as the "PV impermeability theorem". At every point there is precisely zero PV flux across isentropic surfaces, even in the presence of diabatic and frictional processes. Considering a control volume bounded by isentropic surfaces above and below, it is found by integrating (11) that the mass-weighted integral of PV (or total PV substance, using the terminology of Haynes and Mclntyre (1990)) cannot change if the lateral boundary moves with the velocity $\mathbf{V}$ in the isentropic layer and heating and friction do not act on the lateral boundary. This is a generalisation of Kelvin's circulation theorem, as discussed by Methven (2015).

This link with circulation motivates a third form of PV equation in a frame following isentropic trajectories (obtained by integrating V). Such an approach is arguably a more natural choice than (8) since we can define the average PV of any such control volume in terms of its circulation divided by mass and therefore PV at any location can be considered as circulation about a point (in the limit that the control volume tends to zero). For the purpose of the present study, there is an additional benefit to considering the evolution of $\mathrm{PV}$ along isentropic trajectories: that form of the $\mathrm{PV}$ equation gives useful new insight into the conditions under which PV can become negative. Rearranging (11) gives an equation for the evolution of PV following isentropic trajectories:

$$
\rho \frac{\tilde{D} P}{D t}=P \nabla \cdot\left(\rho \mathbf{u}_{D}\right)+\nabla \cdot\left(\boldsymbol{\zeta}_{/ /} Q\right)-\nabla \cdot(\mathbf{F} \times \nabla \theta)
$$

where the Lagrangian derivative following the isentropic flow is:

$$
\frac{\tilde{D}}{D t}=\frac{\partial}{\partial t}+\left(\mathbf{u}-\mathbf{u}_{D}\right) \cdot \nabla=\frac{\partial}{\partial t}+\mathbf{V} \cdot \nabla
$$

Whilst at first sight (13) appears more complex than (8), all of the terms on the right hand side of (13) have simple physical interpretations. The first term represents the effects of mass flux divergence from an isentropic layer by diabatic heating. In terms of the impermeability theorem, this is described as the dilution/concentration of PV substance within the isentropic layer by diabatic heating. Crucially, this term is proportional to $P$ and therefore if initially $P>0$, 
then even if $\nabla \cdot\left(\rho \mathbf{u}_{D}\right)<0$ (diabatic mass flux convergence) the PV will approach zero exponentially but cannot change sign.

The second and third terms on the right hand side of (13) represent non-advective fluxes of PV along isentropic surfaces. The third term is associated with friction and is not considered further here. The physical origin of the second term can be understood by noting that the PV is proportional to the projection of the absolute vorticity vector onto $\mathrm{n}$. If, for example, heating in a baroclinic zone acts to bow the sloping isentropic surfaces downwards then this projection will increase on one side of the heating and decrease on the other. The result can be viewed as flux of PV along isentropic surfaces across the heating region. Defining the non-advective PV flux associated with diabatic heating as

$$
J_{N A}=-\zeta_{/ / Q}
$$

its magnitude is not directly related to PV and as such its action can accumulate over time to turn the PV negative.

\subsection{Visualising the two diabatic terms in the PV equation and their relevance for WCBs}

To visualise the first two (diabatic) terms on the right hand side of (13), first note that to the accuracy of the hydrostatic primitive equations the dilution/concentration term can be written

$$
P \nabla \cdot\left(\rho \mathbf{u}_{D}\right)=P \frac{\partial}{\partial z}\left(\frac{\rho Q}{\theta_{z}}\right) .
$$

Therefore, for localised heating in a uniform flow this term takes the usual form of a vertical dipole with positive values below the heating and negative above (e.g. Wernli, 1997). For the second term note that, assuming the shallow atmosphere and hydrostatic approximations are made,

$$
\boldsymbol{\zeta}=\left(\begin{array}{c}
-v_{z} \\
u_{z} \\
f+v_{x}-u_{y}
\end{array}\right) \approx\left(\begin{array}{c}
-\frac{g}{f \theta_{0}} \theta_{x} \\
-\frac{g}{f \theta_{0}} \theta_{y} \\
f+v_{x}-u_{y}
\end{array}\right)
$$

where the final approximate form assumes anelastic thermal wind balance: $\left(u_{z}, v_{z}\right)=\left(-\theta_{y}, \theta_{z}\right) g / f \theta_{0}$. In that case the horizontal component of $\zeta$ can be seen to be anti-aligned with the horizontal temperature gradient, meaning $\zeta_{/ /}$must point directly up the slope of isentropic surfaces. Therefore $J_{N A}(15)$ always acts to transport PV 'down the isentropic slope'. For a given heating field $Q$, the magnitude of $J_{N A}$ is proportional to the horizontal temperature gradient or, equivalently, the vertical wind shear. The orientation of $J_{N A}$ is illustrated schematic in Fig. 5.

Scaling for the typical magnitudes of the two terms in (13) have been obtained by Haynes and McIntyre (1987) and refined by Martínez-Alvarado et al. (2016). Assume length scales for horizontal $(L)$ and vertical $(H)$ wind variations, and horizontal wind speeds $U$. Then the first two terms of the right hand side of (13) will scale as

$$
(1+R o): R o
$$

where $R_{0}=U / f L$ is the Rossby number. On large scales, where $R_{o}$ is small, the dilution/concentration term will dominate and the PV cannot turn negative. On small scales, where $R o$ is larger than one, the non-advective flux term will become important, and negative PV may occur. This result assumes that variations in the heating field occur on the same spatial scale as the flow. To account for the case of smaller-scale heating embedded within a large-scale flow, 
instead suppose the heating field varies in the horizontal over a spatial scale $\mathcal{L}<L$. In that case, the two terms will scale as

$$
(1+R o): R o \frac{L}{\mathcal{L}}
$$

meaning the importance of the non-advective flux term will be enhanced in that case. For low Rossby number flow, the critical heating length-scale where the two terms become comparable is $L=U / f$ which is typically of the order several hundred kilometers.

For the case of heating in a WCB, negative PV values can therefore only arise when the diabatic heating occurs in a baroclinic shear zone and the heating should have structure on scales of several hundred kilometers or less. Typically WCB heating occurs on these scales since it represents the main ascending air stream of a baroclinic wave, in an elongated structure aligned with the surface cold front. The heating maximum is usually located beneath and equatorward of the jet stream maximum. This setup is illustrated schematically in Fig. 5 in which $\mathbf{J}_{N A}$ is oriented so as to flux PV down the isentropic slope and away from the jet core. The result will be a dipole structure on the isentropic surface, with low or negative PV on the side of the heating nearest the jet and positive values equatorward of this (Fig. 5). Since negative PV is associated with strong anticyclonic shear, the action of the non-advective flux is to increase the shear on the jet stream and, as we shall see, the maximum wind.

As a further consequence of utilising the flux form of the PV equation, it is clear that the area-average value of $P V$, integrated over an area of an isentropic surface encompassing the region of heating, cannot become negative (area-average PV is only diluted/concentrated), whereas smaller-scale dipoles within the heating region may exhibit negative PV values. Another way to see this result is to integrate (11) directly over any region with $J_{N A}=0$ on its boundary, showing that the integrated PV within such a region evolves as if $\mathrm{J}_{N A}$ were completely absent (Methven, 2015). This dynamical constraint is not so obvious from the full Lagrangian form of the PV equation.

\section{5 | EVOLUTION TO A STATE WITH NEGATIVE PV EXPLAINED WITH THE FRONTAL-GEOSTROPHIC EQUATIONS}

To investigate the structure of diabatically-generated PV anomalies more quantitatively, the frontal-geostrophic model is used. The aim is to elucidate how the nonlinear response to heating is shaped by the two diabatic terms in the isentropic PV equation and in particular how the structure and magnitude of these terms change as the PV tends towards zero. Two simulations are presented. The first is the simplest possible setup, that of a localised region of heating in a zone with uniform baroclinicity. The second case puts the same heating field into a more realistic setup including a sloping tropopause structure and associated jet stream. The aim is to mimic a typical baroclinic wave situation where the WCB flowing northwards in the warm sector is below and just ahead of an advancing upper-level trough.

\section{1 | Case 1: Localised heating in a uniform baroclinic zone}

The first case uses the strong shear setup from Chagnon and Gray (2009). The Boussinesq approximation is made (i.e. $\tilde{\rho}(z)=\rho_{0}$ and $\tilde{\theta}(z)=\theta_{0}$, using the notation introduced in App. A), and the initial state is a uniform baroclinic zone in 
thermal wind balance:

$$
\begin{aligned}
m & =-f y+\wedge z \\
\theta & =\frac{\theta_{0}}{g}\left(N^{2} z-f \wedge y\right)
\end{aligned}
$$

Physical constants used are $f=10^{-4} \mathrm{~s}^{-1}, N=10^{-2} \mathrm{~s}^{-1}, \Lambda=0.5 \times 10^{-2} \mathrm{~s}^{-1}, \theta_{0}=300 \mathrm{~K}, g=10 \mathrm{~ms}^{-2}$ and $\rho_{0}=1.2 \mathrm{~kg} \mathrm{~m}^{-3}$. This initial state has a uniform PV: $P=\left(N^{2}-\Lambda^{2}\right) \theta_{0} f /\left(\rho_{0} g\right)=0.19 \mathrm{PVU}$. The heating field used is a Gaussian profile:

$$
Q=Q_{0} \exp \left(\left(\frac{y-y_{0}}{2 \sigma_{y}}\right)^{2}+\left(\frac{z-z_{0}}{2 \sigma_{z}}\right)^{2}\right)
$$

with parameters $Q_{0}=10 \mathrm{Kday}^{-1}, z_{0}=5 \mathrm{~km}, \sigma_{y}=200 \mathrm{~km}$ and $\sigma_{z}=2 \mathrm{~km}$.

For this initial state the two source terms of the isentropic PV tendency (13) can be computed analytically:

$$
\begin{aligned}
P \nabla \cdot\left(\rho \mathbf{u}_{D}\right) & =f\left(1-\frac{\Lambda^{2}}{N^{2}}\right) \frac{\partial Q}{\partial z} \\
\nabla \cdot \mathbf{J}_{N A} & =-\left.\Lambda \frac{\partial Q}{\partial y}\right|_{\theta}
\end{aligned}
$$

These expressions make it clear how the dilution/concentration term (23) is proportional to $P$ and therefore tends to zero as the PV becomes small, which occurs as the vertical wind shear $\wedge$ increases towards the buoyancy frequency $N$. The non-advective flux term (24), in contrast, increases with $\wedge$.

The initial wind and meridional circulation for this setup are shown in Fig. 6a. The meridional circulation in balance with the heating consists of rising motion in the centre of the domain and descent elsewhere (blue contours). The rising motion is not exactly vertical, but is tilted slightly towards the slope of the isentropic surfaces. The simulation runs for 18 hours before the PV turns negative. The final state wind field is shown in Fig. 6c. The isentropic surfaces (brown contours) have bowed downwards inside the heating region and the wind field (green contours) is characterised by anticyclonic shear above the heating and cyclonic shear below. The diabatically-produced PV consistently exhibits a nearly vertical dipole with reduced PV above the heating and increased PV below. The PV dipole is, however, tilted towards the cold side of the heating and the negative PV first appears on this side of the heating, not directly above it.

To interpret this response to heating in terms of the theory presented in Sec. 4, Figs. 6b and 6d show the two source terms of the isentropic PV tendency (13). The dilution term (blue contours) exhibits a vertical dipole at the initial time, whereas the non-advective flux term represents a flux down the isentropic surfaces. The magnitudes of the two terms change substantially during the simulation, with the non-advective flux growing at the expense of the dilution (Fig. $6 \mathrm{~d}$ ). At the point where the PV first turns negative, the dilution term has become zero and the PV tendency is entirely due to the non-advective flux.

This case illustrates a number of features discussed in Sec. 4. In the absence of the non-advective flux term, the dilution produces a vertical dipole of PV associated with the mass flux across the isentropic surfaces. However, this term is unable to turn the PV negative. In contrast, the non-advective flux term acts to transport PV down the isentropic slope and is associated with the tilting of the isentropic surface across the heating region. The change in the relative magnitudes of the two terms is due in a large part to the distortion of the isentropic surfaces (and so a change in the projection of $\zeta$ onto them), rather than a change in the total PV tendency. 


\section{2 | Case 2: A more realistic jet stream example}

The second illustration introduces some complexity by putting the heating field in a localised baroclinic zone. An initial PV profile representing a localised jet stream is constructed, with $P=P_{T}$ in the troposphere, $P=P_{S}$ in the stratosphere and a smooth transition between the two across the tropopause at height

$$
z=Z_{T}+\frac{\Delta Z_{T}}{2} \operatorname{erf}\left(\frac{y-Y_{J}}{\Delta Y_{J}}\right)
$$

This initial PV field is inverted with $u=0$ at the lateral boundaries and $\theta=$ constant at the top and bottom, as described in App. A.

Physical constants used are the same as before, with the initial condition parameters $P_{T}=0.26 \mathrm{PVU}, P_{S}=2.91 \mathrm{PVU}$, and $Z_{T}=8 \mathrm{~km}, \Delta Z_{T}=3 \mathrm{~km}, Y_{J}=300 \mathrm{~km}$ and $\Delta Y_{J}=100 \mathrm{~km}$. The transition across the tropopause is isotropic in the scaled coordinates $(y, 100 z)$ over a distance of $50 \mathrm{~km}$ in the horizontal and $500 \mathrm{~m}$ in the vertical. The heating profile is similar to case 1 , but modified to be a cosine-squared profile to avoid heating above the tropopause. This initial setup is shown in Fig. 7a.

Figure 7c shows the wind, potential temperature and PV at the final time for this simulation. This case ran for 21 hours before the PV turned negative. As in case 1 the heating has generated a PV dipole, with low PV values on the upper-poleward side of the heating region and positive values below the heating. In addition, the cross-frontal secondary circulation associated with diabatic heating has acted to advect the tropopause poleward, shifting the jet stream with it. At low levels, the wind speed maximum is shifted equatorward, despite the poleward-shifted upper-tropospheric jet, due to the low-level diabatically-generated positive PV anomaly.

The remaining panels in Fig. 7 show the isentropic decomposition of the isentropic PV tendency equation. As in case 1 , the dilution term exhibits a vertical dipole but the non-advective flux term represents a flux down the isentropic surfaces (panels (b) and (d)). These two terms change substantially during the simulation, with the non-advective flux growing in magnitude at the expense of the dilution.

When viewed on an isentropic surface below the jet maximum, the impact of the diabatic heating is to produce a PV dipole with the negative PV closest to the jet core. In practice the negative PV could be expected to be strung out along the anticyclonic side of the jet axis. Negative PV corresponds to a stronger anticyclonic shear and the jet maximum will be stronger as a result of both the sharpening of the tropopause PV gradient and the non-advective PV flux. Both of these effects are not accounted for in the quasi-geostrophic model, but are incorporated in the semi-geostrophic (and frontal-geostrophic) model.

\section{6 | THE ORIGIN OF THE NEGATIVE PV IN THE OBSERVED CASE}

In Sec. 3 evidence was presented for observations of negative PV. Theoretical considerations of the mechanisms by which PV can turn negative were explored in Sec. 4, and illustrated in Sec. 5 with the 2D frontal-geostrophic model, which is the simplest model containing the essential dynamics of the problem. In this final section attention is returned to the NAWDEX case study of Sec. 3 and its representation in a numerical weather prediction model. The aim is to relate insight from the theoretical developments to identify the physical processes responsible for the negative PV observed. 


\section{1 | Simulation of NAWDEX IOP4 case}

Figure 8 shows maps from a 36 hour forecast valid at $12 Z$ on 27 September 2016. This is the time of the research flights of Sec. 3. Panel (a) shows the full PV field at $8.1 \mathrm{~km}$ and should be compared to the PV derived from dropsonde observations (Fig. 3) along section AB in Fig. 1d. As in Fig. 3, there are two regions of negative PV: one to the north of the stratospheric intrusion due north of the UK, and one to the south of the stratospheric intrusion to the west of the UK.

To investigate whether the negative PV was present in the initial state or is been generated by the model during the 36 hours of the simulation, panel (b) shows the advection-only PV tracer at the same altitude (see Sec. 2.1). The advection-only tracer is remarkably similar to the full PV field in many respects, such as the north-south filament of high PV air over central Europe captured with great accuracy. The most notable difference between the fields, however, is the almost complete absence of the negative PV in the advection-only tracer. Neither of the observed negative PV regions are present. The negative PV values have therefore been generated by the model during the simulation. Panel (c) shows the sum of the PV tracers for all of the physical processes and panel (d) shows the sum of the PV tracers associated with the dynamical core (see Sec. 2.1). It is evident that both regions of negative PV are associated with the parametrized physical processes, rather than non-conservation by the dynamical core.

The negative PV values to the south of the stratospheric intrusion have a banded di-polar structure aligned with the flow and with relatively narrow spatial scales $(\mathrm{O}(100 \mathrm{~km}))$. This is expected since this region lies in the strong baroclinic zone beneath the jet maximum and hence the non-advective PV flux will play a dominant role. The sign of the anomalies is such that PV has been fluxed 'down the isentropic slope' by the heating: the negative PV anomalies are closest to the jet core for each band of heating, as illustrated schematically in Fig. 5 and in the frontal-geostrophic model in Figs. 6 and 7.

In order to understand which physical processes are important for the generation of the negative PV in this case, Fig. 9 presents the individual PV tracers for the four most important parametrization schemes. In the region of the jet core to the south of the stratospheric intrusion, the tracers show that the negative PV arises due to heating in shear through a combination of the convection scheme (panel (d)) and the large-scale rain scheme (panel (b)). This is consistent with the action of mid-level WCB heating in the baroclinic shear below the jet giving rise to a non-advective PV flux down the isentropic slope towards the south. There are contributions to the negative PV on the north side of the stratospheric intrusion from all four of the schemes shown. As found by Chagnon et al. (2013), and shown to be systematic by Saffin et al. (2017), the radiation scheme tracer (panel (a)) is positive within troughs and negative in ridges. This is likely a result of long wave cooling spike associated with the jump in humidity at the tropopause (Forster and Wirth, 2000).

By following the negative PV features backwards in time on isentropic surfaces (shown in Fig. S2) it can be seen that the feature to the north has developed in the last 3 hours of the simulation, whereas the bands of negative PV to the south have developed more gradually over the previous 24 hours, following the flow around the ridge from the western flank above the WCB. The banded features appear to develop as elongated features, rather than resulting from a stretching by the flow.

\section{2 | Observational evidence for the banding in PV south of the jet core}

As noted above, the negative PV to the south of the stratospheric intrusion has a clear banded structure aligned with the jet. Unfortunately the dropsonde data from the FAAM aircraft presented in Figs. 2 and 3 does not extend far enough south to test whether this model-generated feature is realistic in its position and structure. However, as shown in Fig. 1, two other coordinated research flights took place at the same time. In this section the in situ wind data from the HALO aircraft is used to look for evidence of these features in the observations of horizontal wind shear. 
The portion of the HALO flight track analysed here is the north-south section along $29 \mathrm{~W}$ (see Fig. 1d, green line CD). This lies further west than the FAAM flight track, but is better placed to observe the negative PV structures. The flight track is shown in Figs. 8 and 9 by the dashed black line. A cross section of the simulated PV field along this section is shown in Fig. 10a. The banded structure is clearly present along the southern half of the section CD and is associated with shallow ripples in the potential temperature surfaces (grey contours). The sum of the physics PV tracers show that the whole structure arises from the action of diabatic processes during the course of the simulation (Fig. 10b). The diabatic PV changes near point $C$ are typical of the action of turbulent mixing and long wave radiative cooling in a deep trough, eroding the PV underneath and enhancing PV on the stratospheric side of the tropopause (as shown in Saffin et al. (2017)).

To assess the realism of the simulated PV banding, Fig. 10c compares the vertical component of absolute vorticity computed from the in situ wind observations of the HALO aircraft and vorticity from the MetUM simulation. The observational estimate has been obtained using the same methodology as in Fig. 4. The high-frequency (16 Hz) wind data is first smoothed with a 20 s Gaussian kernel smoothing, and then the local across-jet wind shear is computed using a finite difference. The resulting field (gray line) has substantial variability on $\mathrm{O}(20 \mathrm{~km})$ spatial scales that cannot be resolved by the model (grid spacing $17 \mathrm{~km}$ ) although it is very well resolved by the measurements (sample spacing $\approx 2 \mathrm{~m}$ ). For comparison with the model data, an additional 10 minute running mean filter has been used to remove the variability (blue line). The aircraft speed was $160 \mathrm{~ms}^{-1}$ throughout this section so that a 10 minute mean corresponds to a spatial distance of $96 \mathrm{~km}$ ( $\approx 6$ grid points).

The smoothed observed vorticity shows some similarity to the MetUM data (red line). In particular, there is evidence of variability of the vorticity field on a similar spatial scale ( $\approx 200 \mathrm{~km}$ between vorticity peaks). The green arrows indicate two of the bands of positive diabatically-generated PV in the simulation and are clearly associated with areas of more positive absolute vorticity in the observations. Around one degree of latitude north of each of these positions lie corresponding minima in the absolute vorticity.

This case study suggests the representation of the banded negative PV structures in the model may be realistic on the scales that it can resolve. However, it also shows that there is additional variability on shorter length-scales. For instance, the pronounced oscillation in wind shear between $48.5 \mathrm{~N}$ and $50 \mathrm{~N}$ with a wavelength around $50 \mathrm{~km}$ could be due to narrow bands of heating in the shear environment and non-advective PV flux, or it could be a signature of inertia gravity wave activity on scales too small to be resolved by the model. Future work will aim to assess the representation of these features in higher-resolution versions of this model.

\section{7 | DISCUSSION AND CONCLUSIONS}

High resolution observations from aircraft and dropsondes during the NAWDEX field experiment have revealed that Ertel potential vorticity (PV) can be negative on the flanks of the jet stream. Since the background PV is everywhere positive in the extratropical Northern Hemisphere, negative PV cannot arise through adiabatic, frictionless motion as $\mathrm{PV}$ is conserved along trajectories. The conditions under which diabatic heating can act to turn the PV negative are examined and it is shown that although the convergence of diabatic mass transport in the upper troposphere, associated with air crossing isentropic surfaces in regions of heating, results in the decrease of PV through dilution, this process cannot make the PV change sign. This deduction is a consequence of the PV impermeability theorem of Haynes and Mclntyre (1987, 1990).

The negative PV must arise through the non-advective PV flux which creates quasi-horizontal PV dipoles whenever there is heating in the presence of vertical wind shear. The non-advective PV flux is directed along isentropic surfaces 
and is always oriented 'down the isentropic slope' due to thermal wind balance. The structure of baroclinic waves is such that air moving polewards in the warm conveyor belts (WCBs) of cyclones, ascending and releasing latent heat in the process, is typically situated just to the east of the leading edge of an upper level trough - a consequence of the westward phase tilt necessary for the growth of baroclinic waves (Heifetz et al., 2004). Therefore the diabatic heating extending along the WCB often occurs beneath the jet stream in an environment with strong vertical wind shear. In these situations the non-advective PV flux transports PV away from the jet core, with the consequence that PV is more negative on its equatorward flank, resulting in increased anticyclonic wind shear and jet maximum winds, described here as a "sharpening" of the jet stream.

Idealised frontal-geostrophic simulations are presented with prescribed heating, showing that the magnitude of the PV dipole increases until the negative lobe reaches zero PV, at which point the frontal-geostrophic model ceases to be invertible. However, the non-advective PV flux mechanism is completely general and there is no bound on how negative the PV could become. Scaling the isentropic PV equation shows that the ratio of the non-advective PV flux to the diabatic mass flux convergence term scales as Ro/(1+Ro) where Ro is the Rossby number (Martínez-Alvarado et al., 2016). So on synoptic scales (Ro < 1), the non-advective term is expected to be relatively small, while on mesoscales and smaller (Ro » 1) the terms are of equal importance. This helps to explain why negative PV is typically found in strips aligned with the jet stream. In convective-permitting simulations of NAWDEX cases (Oertel et al., in review) each local maximum in heating generates a PV dipole (as described in Chagnon and Gray (2009)) but a consequence of the synoptic scale vertical wind shear is that all the PV dipoles must be aligned with the negative side facing the jet stream core. A direct result of the common orientation is an upscale influence of diabatic processes on the flow field, felt through the integral effects of the dipoles. Note also that since an extended dipole has a much shorter-range influence on the flow than a monopole (through the elliptic nature of PV inversion) the influence of the diabatic processes is expected to be localised near the heating (mesoscale in the cross-jet direction).

The diabatically-generated PV dipoles can occur in multiple bands, as shown in Fig. 8. This would be the natural result of parallel bands in heating (e.g., rainbands) in an environment of larger-scale vertical wind shear. However, the leading question is why the heating should be generated in bands. One plausible explanation is that both the bands in heating and the PV dipoles arise together as a consequence of conditional symmetric instability (CSI). The theory of symmetric instability is framed in an idealised situation where the PV of a baroclinic shear parallel flow is initially uniform (Hoskins, 1974). If the PV is negative, then the flow is unstable with respect to slantwise motions and slantwise circulations (mesoscale in the cross-flow direction) can grow. However, Griffiths (2008) has shown that even if the $\mathrm{PV}$ is slightly positive the same structures can be readily excited (modal structures are continuous across the stability boundary), although they do not grow exponentially, and are interpreted as inertia-gravity wave motions. In the ridge shown in Fig. 8, the PV would be near zero across an extensive region, even in the absence of diabatic processes (as shown by the advection-only PV tracer), but in this location humidity saturation can be expected allowing the possibility that saturation moist PV is negative and the necessary conditions for CSI are met (Bennetts and Hoskins, 1979). So even if saturation does not occur everywhere, the slantwise structures can grow. The model cross-section (Fig. 10b) shows clearly the slantwise nature of the PV bands (the aspect ratio of the plot is approximately 100:1 so a feature at an angle of 45 degrees has scales $L / H \sim N / f$ ). So we hypothesize that the multiple bands emerge due to CSI, but then the consequence of the non-advective PV flux is that the moist dynamics must lead to negative PV if the heating is sustained for long enough. The negative PV has a signature of negative absolute vorticity (not static instability) which has often been associated with strong jet streams. Although some authors describe these structures as regions of "inertial instability" (e.g., Schultz and Knox (2007)), they arise in a highly baroclinic environment with strong horizontal pressure gradients (not the conditions required for pure inertial instability). Instead, we hypothesise the structures with negative absolute vorticity form as a consequence of finite amplitude CSI (rather than drivers of instability), as originally 
argued by Thorpe and Clough (1991) from aircraft observations of slantwise circulations above lower tropospheric cold fronts.

There are several consequences of our findings for forecasting the jet stream strength and clear air turbulence with ramifications for the aviation industry. Firstly, the systematic structure of the PV dipoles generated by heating in vertical wind shear, means that diabatic processes must contribute to extremes in jet stream strength. Also the position of the jet core is altered by advection of the tropopause by additional divergent outflow associated with heating. Therefore diabatic processes must influence the prediction of the jet stream, essential to inter-continental flight routing, and uncertainty in the representation of diabatic processes in forecast models may be limiting predictive skill (e.g., Rodwell et al. (2013)).

Secondly, inertial instability (in the sense of regions diagnosed with negative absolute vorticity) has been postulated as one of the numerous sources of clear air turbulence (CAT) experienced by airliners. It has recently been shown that many different CAT indicators tend to exhibit increases in the frequency of extreme events in future climate simulations (Williams, 2017; Storer et al., 2017). This is likely in part due to increased vertical wind shear in the time mean (Lee et al., 2019). However, out of the many different CAT indicators, the ones which show the biggest increase in extremes are not the ones that measure vertical wind shear but rather strong (anticyclonic) horizontal wind shear (see Fig. 2 of Williams, 2017). This could suggest that it is the diabatic generation of negative PV within the jet stream that increases the occurrence of CAT and therefore changes to latent heat release associated with increasing moisture in a warmer climate may contribute to the increased probability of encountering turbulence.

Research underway is investigating the influence of climate change on the jet stream mediated through changes in latent heat release in shear. Since saturation vapour pressure increases in a warmer world, greater latent heat release is expected for the same vertical motion on saturated ascent. In a situation where the vertical wind shear is the same, then a greater non-advective PV flux and sharpening of the jet stream might be expected. However, the response of the jet stream (wind shear) and storm track (vertical motion) to climate change is uncertain and future work will investigate the ramifications of the mechanism identified here for projected changes in extreme jet stream winds.

\section{ACKNOWLEDGEMENTS}

Many international institutions contributed to the NAWDEX field campaign. The authors would like particularly to thank the UK Met Office for funding the flights of the FAAM aircraft and the dropsondes it released throughout NAWDEX. The HALO flights were funded by the DLR (project "Klimarelevanz von atmosphärischen Spurengasen, Aerosolen und Wolken" KliSAW), the German Science Foundation (DFG; within SPP1294 HALO and SFB/TRR165 Waves to Weather) and the European Space Agency (ESA, provided funds related to the preparation of EarthCARE (EPATAN, contract No. 4000119015/16/NL/CT/gp)). BH acknowledges National Capability funding from the Natural Environment Research Council through the National Centre for Atmospheric Science.

\section{CONFLICT OF INTEREST}

\section{A | THE FRONTAL-GEOSTROPHIC EQUATIONS}

The equation set mentioned in Sec. 2.2 and used in Sec. 5 is based on that in Hoskins and James (2014), Sec. 15.2, with the addition of diabatic heating and friction included. For completeness an outline derivation is presented here. For notation assume a zonally-symmetric setup oriented in the $x$-direction and make the $f$-plane and anelastic approximations. The 
momentum, thermodynamic and mass conservation equations are then:

$$
\begin{aligned}
\frac{D m}{D t} & =F \\
\frac{D v}{D t} & =-\left(\frac{p^{\prime}}{\tilde{\rho}}\right)_{y}-f u \\
\frac{D w}{D t} & =-\left(\frac{p^{\prime}}{\tilde{\rho}}\right)_{z}+\frac{g \theta^{\prime}}{\tilde{\theta}} \\
\frac{D \theta}{D t} & =Q \\
(\rho v)_{y}+(\rho w)_{z} & =0 .
\end{aligned}
$$

Here $m=-f y+u$ is the absolute zonal momentum with $f$ the (constant) Coriolis parameter and $u$ the zonal wind, $v$ and $w$ are the meridional and vertical wind components, $F$ is friction and $Q$ is heating. The anelastic approximation assumes fixed reference profiles of pressure $p$, density $\rho$ and potential temperature $\theta$ which are a function of $z$ only, indicated here by tildes and deviations are indicated by primes. The advective derivative used throughout represents advection in the meridional plane only due to the assumed zonal symmetry:

$$
\frac{D}{D t}=\frac{\partial}{\partial t}+v \frac{\partial}{\partial y}+w \frac{\partial}{\partial z}
$$

The PV for this system is

$$
P=\left(m_{z} \theta_{y}-m_{y} \theta_{z}\right) / \tilde{\rho}
$$

which satisfies

$$
\tilde{\rho} \frac{D P}{D t}=F_{z} \theta_{y}-F_{y} \theta_{z}+m_{z} Q_{y}-m_{y} Q_{z}
$$

as can be seen from manipulating (26) and (29).

The frontal-geostrophic approximation replaces (27) and (28) by estimates of $v$ and $w$ obtained as follows. Assuming the advective derivatives in (27) and (28) are small implies that the zonal flow must be in thermal wind balance (TWB):

$$
m_{z}=-\frac{g}{f \tilde{\theta}} \theta_{y}
$$

If TWB holds then the time derivatives in (26) and (29) can be eliminated to find

$$
v_{z} m_{y}+w_{z} m_{z}+\frac{g}{f \tilde{\theta}}\left(v_{y} \theta_{y}+w_{y} \theta_{z}\right)=F_{z}+\frac{g}{f \tilde{\theta}} Q_{y}
$$

Finally, using mass conservation to write the meridional circulation in terms of a streamfunction for the secondary "cross-frontal" circulation: $(v, w)=\left(-\partial_{z} \varphi, \partial_{y} \varphi\right) / \tilde{\rho}$,

$$
\varphi_{y y} \frac{g}{f \tilde{\theta}} \theta_{z}+\varphi_{y z}\left(m_{z}-\frac{g}{f \tilde{\theta}} \theta_{y}\right)-\varphi_{z z} m_{y}+\frac{1}{\tilde{\rho}} \frac{d \tilde{\rho}}{d z}\left(\varphi_{z} m_{y}-\varphi_{y} m_{z}\right)=\tilde{\rho}\left(F_{z}+\frac{g}{f \tilde{\theta}} Q_{y}\right)
$$

This is the Sawyer-Eliassen equation for the case with heating and friction but no geostrophic forcing. The final term on 
the left hand side is due to vertical variations in the anelastic approximation reference state profiles and vanishes if the more restrictive Boussinesq approximation is made.

Given initial $m$ and $\theta$ fields, (36) is an elliptic equation which can be solved for $\varphi$ provided $P>0$. This is done numerically on the domain shown in Figs. 6 and 7 using a successive over-relaxation Gauss-Seidel method with boundary conditions $v=0$ at meridional boundaries and $w=0$ at the upper and lower boundaries. The resulting meridional flow is then used to directly integrate (26) and (29) forward one time step. An alternative solution procedure is to use $P$ as a prognostic variable via (33), invert it to find the unique $m$ and $\theta$ fields in TWB satisfying (32), and then integrate the PV conservation equation (33) forward one timestep. The former time stepping method is simpler to implement and found to be sufficient for the illustrations presented in this paper. An inversion of $P$ to find $m$ and $\theta$ is however performed to determine the initial state used in Sec. 5.2 and this done numerically with a similar iterative over-relaxation method with boundary conditions $u=0$ at meridional boundaries and $\theta^{\prime}=0$ at the upper and lower boundaries.

\section{REFERENCES}

Bennetts, D. A. and Hoskins, B. J. (1979) Conditional symmetric instability - a possible explanation for frontal rainbands. Q. J. Roy. Meteor. Soc., 105, 945-962.

Chagnon, J. M. and Gray, S. L. (2009) Horizontal potential vorticity dipoles on the convective storm scale. Q. J. Roy. Meteor. Soc., 135, 1392-1408.

Chagnon, J. M., Gray, S. L. and Methven, J. (2013) Diabatic processes modifying potential vorticity in a North Atlantic cyclone. Q. J. Roy. Meteor. Soc., 139, 1270-1282.

Danielsen, E. F., Hipskind, R. S., Gaines, S. E., Sachse, G. W., Gregory, G. L. and Hill, G. F. (1987) Three-dimensional analysis of potential vorticity associated with tropopause folds and observed variations of ozone and carbon monoxide. J. Geophys. Res.: Atmos., 92, 2103-2111.

Danielsen, E. F. and Mohnen, V. A. (1977) Project Dustorm report: Ozone transport, in situ measurements, and meteorological analyses of tropopause folding. J. Geophys. Res., 82, 5867-5877.

Edwards, J. M. and Slingo, A. (1996) Studies with a flexible new radiation code. I: Choosing a configuration for a large-scale model. Q. J. Roy. Meteor. Soc., 122, 689-719.

Eichelberger, S. J. and Hartmann, D. L. (2007) Zonal jet structure and the leading mode of variability. J. Clim., 20, 5149-5163.

Euler, C., Riemer, M., Kremer, T. and Schömer, E. (2019) Lagrangian description of air masses associated with latent leat release in tropical storm Karl (2016) during extratropical transition. Mon. Wea. Rev., 147, 2657-2676.

Forster, C. and Wirth, V. (2000) Radiative decay of idealized stratospheric filaments in the troposphere. J. Geophys. Res.: Atmos., 105, 10169-10184.

Grams, C. M. and Archambault, H. M. (2016) The key role of diabatic outflow in amplifying the midlatitude flow: A representative case study of weather systems surrounding western North Pacific extratropical transition. Mon. Wea. Rev., 144, 3847-3869.

Grams, C. M., Wernli, H., Böttcher, M., Čampa, J., Corsmeier, U., Jones, S. C., Keller, J. H., Lenz, C.-J. and Wiegand, L. (2011) The key role of diabatic processes in modifying the upper-tropospheric wave guide: A North Atlantic case-study. Q. J. Roy. Meteor. Soc., 137, 2174-2193.

Gray, S. L. (2006) Mechanisms of midlatitude cross-tropopause transport using a potential vorticity budget approach. J. Geophys. Res.: Atmos., 111. 
Gray, S. L., Dunning, C. M., Methven, J., Masato, G. and Chagnon, J. M. (2014) Systematic model forecast error in Rossby wave structure. Geophys. Res. Lett., 41, 2979-2987.

Gregory, D. and Rowntree, P. R. (1990) A mass flux convection scheme with representation of cloud ensemble characteristics and stability-dependent closure. Mon. Wea. Rev., 118, 1483-1506.

Griffiths, S. D. (2008) The limiting form of inertial instability in geophysical flows. J. Fluid Mech., 605, 115-143.

Harvey, B., Methven, J. and Ambaum, M. H. P. (2018) An adiabatic mechanism for the reduction of jet meander amplitude by potential vorticity filamentation. J. Atmos. Sci., 75, 4091-4106.

Harvey, B. J., Methven, J. and Ambaum, M. H. P. (2016) Rossby wave propagation on potential vorticity fronts with finite width. J. Fluid Mech., 794, 775-797.

Haynes, P. H. and Mclntyre, M. E. (1987) On the evolution of vorticity and potential vorticity in the presence of diabatic heating and frictional or other forces. J. Atmos. Sci., 44, 828-841.

- (1990) On the conservation and impermeability theorems for potential vorticity. J Atmos. Sci., 47, 2021-2031.

Heifetz, E., Bishop, C. H., Hoskins, B. J. and Methven, J. (2004) The counter-propagating Rossby-wave perspective on baroclinic instability. I: Mathematical basis. Q. J. Roy. Meteor. Soc., 130, 211-231.

Hoinka, K. P. (1999) Temperature, humidity, and wind at the global tropopause. Mon. Wea. Rev., 127, 2248-2265.

Hoskins, B. and Berrisford, P. (1988) A potential vorticity perspective of the storm of 15-16 October 1987. Weather, 43, 122129.

Hoskins, B. J. (1971) Atmospheric frontogenesis models: Some solutions. Q. J. Roy. Meteor. Soc., 97, 139-153.

- (1974) The role of potential vorticity in symmetric stability and instability. Q. J. Roy. Meteor. Soc., 100, 480-482.

- (1975) The geostrophic momentum approximation and the semi-geostrophic equations. J. Atmos. Sci., 32, $233-242$.

Hoskins, B. J. and James, I. N. (2014) Fluid Dynamics of the Mid-Latitude Atmosphere. Wiley and Sons.

Joos, H. and Forbes, R. M. (2016) Impact of different IFS microphysics on a warm conveyor belt and the downstream flow evolution. Q. J. Roy. Meteorol. Soc., 142, 2727-2739.

Lee, S. H., Williams, P. D. and Frame, T. H. A. (2019) Increased shear in the North Atlantic upper-level jet stream over the past four decades. Nature, $\mathbf{X X X}, \mathbf{X X X}$.

Lock, A., Brown, A. R., Bush, M. R., Martin, G. M. and Smith, R. N. B. (2000) A new boundary layer mixing scheme. Part I: Scheme description and single-column model tests. Mon. Wea. Rev., 128, 3187-3199.

Madonna, E., Wernli, H., Joos, H. and Martius, O. (2014) Warm conveyor belts in the ERA-interim dataset (1979-2010). Part i: Climatology and potential vorticity evolution. J. Clim., 27, 3-26.

Martínez-Alvarado, O., Gray, S. L. and Methven, J. (2016) Diabatic processes and the evolution of two contrasting summer extratropical cyclones. Mon. Wea. Rev., 144, 3251-3276.

Martínez-Alvarado, O., Joos, H., Chagnon, J., Boettcher, M., Gray, S. L., Plant, R. S., Methven, J. and Wernli, H. (2014) The dichotomous structure of the warm conveyor belt. Q. J. Roy. Meteor. Soc., 140, 1809-1824.

Methven, J. (2015) Potential vorticity in warm conveyor belt outflow. Q. J. Roy. Meteor. Soc., 141, 1065-1071.

Nielsen-Gammon, J. W. (2001) A visualization of the global dynamic tropopause. Bull. Amer. Meteor. Soc., 82, 1151-1168. 
Oertel, A., Boettcher, M., Joos, H., Sprenger, M. and Wernli, H. (in review) Potential vorticity structure of embedded convection in a warm conveyor belt and its relevance for the large-scale dynamics. Weather Clim. Dynam. Discuss.

Pasch, R. J. and Zelinsky, D. A. (2016) Tropical cyclone report: Tropical Storm Karl. National Hurricane Center, 12, 1-17.

Pyle, M. E., Keyser, D. and Bosart, L. F. (2004) A diagnostic study of jet streaks: Kinematic signatures and relationship to coherent tropopause disturbances. Mon. wea. Rev., 132, 297-319.

Raymond, D. J. (1992) Nonlinear balance and potential-vorticity thinking at large Rossby number. Q. J. Roy. Meteorol. Soc., 118, 987-1015.

Rodwell, M. J., Magnusson, L., Bauer, P., Bechtold, P., Bonavita, M., Cardinali, C., Diamantakis, M., Earnshaw, P., Garcia-Mendez, A., Isaksen, L. et al. (2013) Characteristics of occasional poor medium-range weather forecasts for Europe. Bull. Amer. Meteor. Soc., 94, 1393-1405.

Saffin, L., Gray, S. L., Methven, J. and Williams, K. D. (2017) Processes maintaining tropopause sharpness in numerical models. J. Geophys. Res., 122, 9611-9627.

Saffin, L., Methven, J. and Gray, S. L. (2016) The non-conservation of potential vorticity by a dynamical core compared with the effects of parametrized physical processes. Q. J. Roy. Meteor. Soc., 142, 1265-1275.

Schäfler, A., Craig, G., Wernli, H. and co authors (2018) The North Atlantic Waveguide and Downstream Impact Experiment. Bull. Amer. Meteor. Soc.

Schultz, D. M. and Knox, J. A. (2007) Banded convection caused by frontogenesis in a conditionally, symmetrically, and inertially unstable environment. Mon. Wea. Rev., 135, 2095-2110.

Shapiro, M. A. (1974) A multiple structured frontal zone-jet stream system as revealed by meteorologically instrumented aircraft. Mon. Wea. Rev., 102, 244-253.

- (1976) The role of turbulent heat flux in the generation of potential vorticity in the vicinity of upper-level jet stream systems. Mon. Wea. Rev., 104, 892-906.

Shapiro, M. A., Hampel, T. and Krueger, A. J. (1987) The Arctic tropopause fold. Mon. Wea. Rev., 115, 444-454.

Storer, L. N., Williams, P. D. and Joshi, M. M. (2017) Global response of clear-air turbulence to climate change. Geophys. Res. Lett., 44, 9976-9984.

Thorpe, A. J. and Clough, S. A. (1991) Mesoscale dynamics of cold fronts: Structures described by dropsoundings in FRONTS 87. Q. J. Roy. Meteor. Soc., 117, 903-941.

Vaughan, G., O'connor, F. M. and Wareing, D. P. (2001) Observations of streamers in the troposphere and stratosphere using ozone lidar. J. Atmos. Chem., 38, 295-315.

Walters, D., Brooks, M., Boutle, I., Melvin, T., Stratton, R., Vosper, S., Wells, H., Williams, K., Wood, N., Allen, T. et al. (2017) The Met Office unified model global atmosphere 6.0/6.1 and JULES global land 6.0/6.1 configurations. Geosci. Model Dev., 10, 1487-1520.

Wernli, H. (1997) A lagrangian-based analysis of extratropical cyclones. II: A detailed case-study. Q. J. Roy. Meteor. Soc., 123, 1677-1706.

Williams, P. D. (2017) Increased light, moderate, and severe clear-air turbulence in response to climate change. Adv. Atmos. Sci., 34, 576-586.

Wilson, D. R. and Ballard, S. P. (1999) A microphysically based precipitation scheme for the UK Meteorological Office Unified Model. Q. J. Roy. Meteor. Soc., 125, 1607-1636. 
Wood, N., Staniforth, A., White, A., Allen, T., Diamantakis, M., Gross, M., Melvin, T., Smith, C., Vosper, S., Zerroukat, M. et al. (2014) An inherently mass-conserving semi-implicit semi-lagrangian discretization of the deep-atmosphere global nonhydrostatic equations. Q. J. Roy. Meteor. Soc., 140, 1505-1520.

Woollings, T., Hannachi, A. and Hoskins, B. (2010) Variability of the North Atlantic eddy-driven jet stream. Q. J. Roy. Meteor. Soc., 136, 856-868.

\section{GRAPHICAL ABSTRACT}

(a)

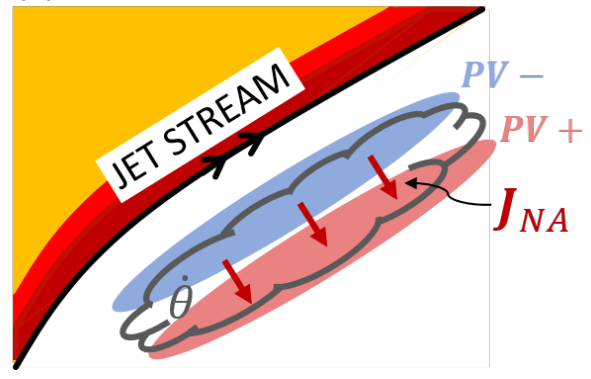

The process by which diabatic heating in warm conveyor belts (WCBs) can act to change the sign of potential vorticity (PV) is studied in observations, theory and models. In the Northern Hemisphere PV is typically positive, and the occurrence of localised regions of negative $\mathrm{PV}$ are evidence of recent diabatic activity. The schematic shows the typical structure of negative PV features associated with latent heating in WCBs. 
(a) WSP 250hPa $26 \mathrm{Sep} 00 \mathrm{Z}$

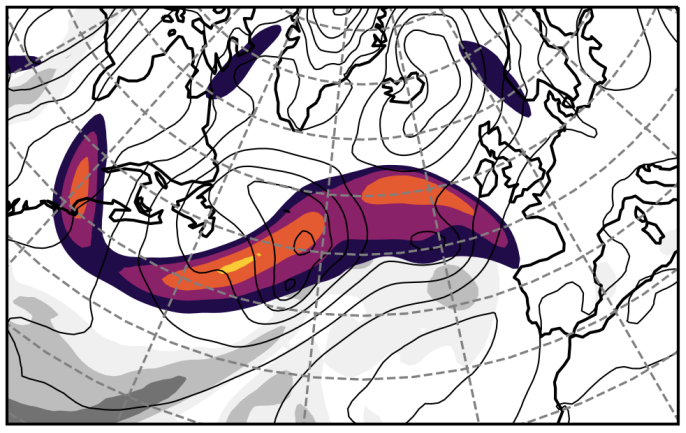

(c) WSP $250 \mathrm{hPa} \mid 27 \mathrm{Sep} 12 \mathrm{Z}$
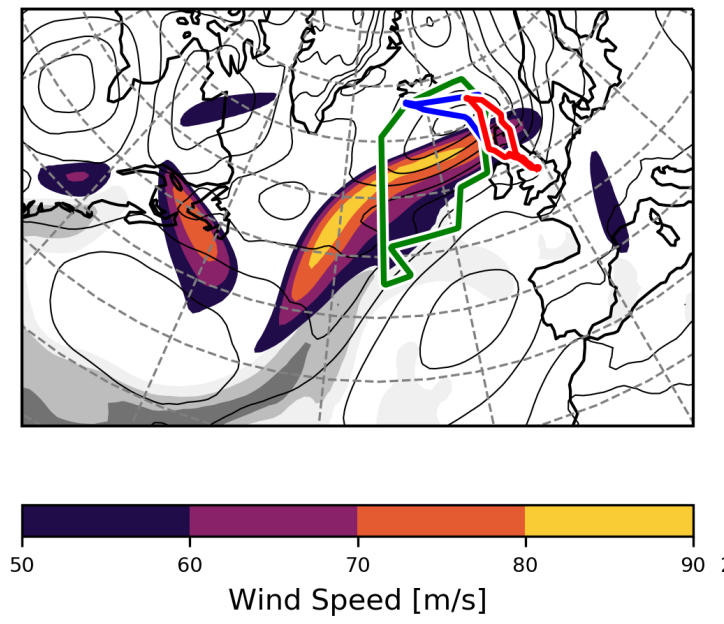

(b) PV 315K | 26 Sep $00 Z$

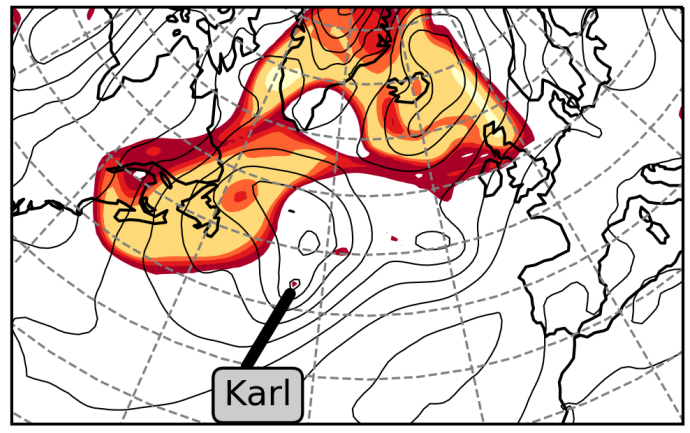

(d) PV 315K | 27 Sep $12 Z$
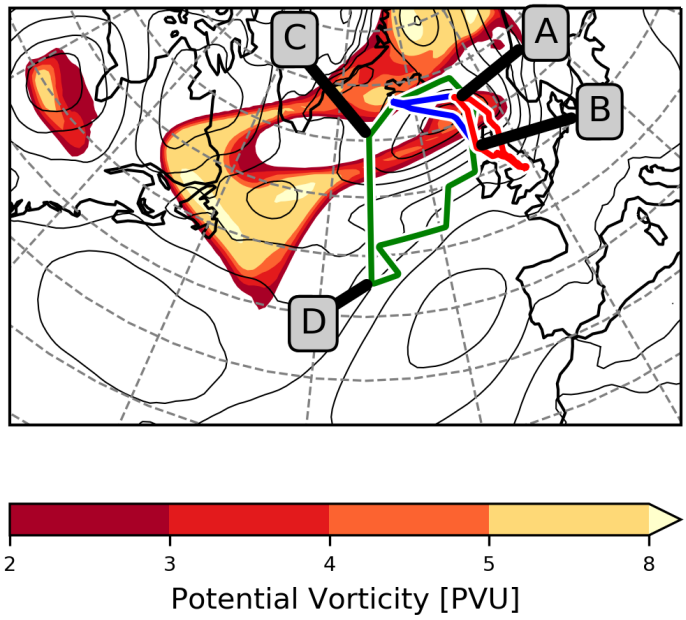

FIGURE 1 Overview of the synoptic evolution at (a, b) $00 Z 26 \mathrm{Sep}$ and (c, d) $12 Z 27$ Sep. Panels (a) and (c) show wind speed at $250 \mathrm{hPa}$ (colours), MSLP (contours) and total column water (grey shading; contoured at intervals of $10 \mathrm{~kg} \mathrm{~m}^{-2}$ starting at $30 \mathrm{~kg} \mathrm{~m}^{-2}$ ). Panels (b) and (d) show potential vorticity on the $315 \mathrm{~K}$ isentropic surface. Also shown in panels (c) and (d) are the flight tracks from the three NAWDEX flights on 27 Sep referred to in the text (green=HALO, blue=DLR-Falcon, red=FAAM). Annotations indicate the position of ex-tropical storm Karl (panel b) and the sections $A B$ of the FAAM flight track used in Figs. 2-4 and CD of the HALO flight track is used in Figs. 8-10 (panel d). Data is from ERA-Interim. 
(a) $u[\mathrm{~m} / \mathrm{s}]$

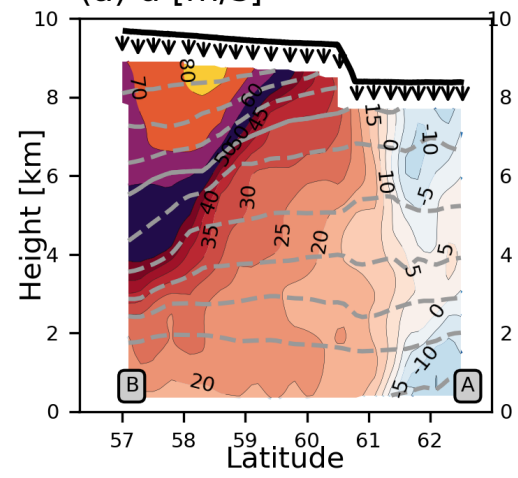

(b) $v[\mathrm{~m} / \mathrm{s}]$

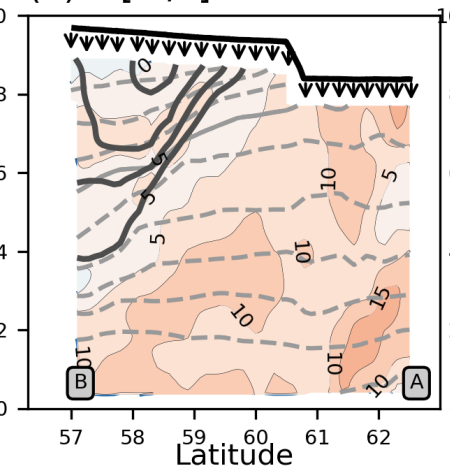

(c) $\mathrm{RH}[\%]$

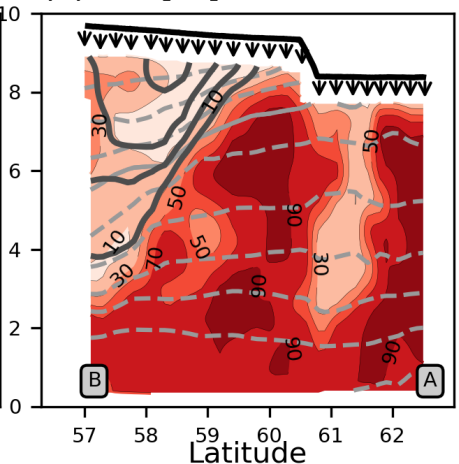

F I G URE 2 Dropsonde data from FAAM flight B981 on 27 Sep: (a) zonal wind, (b) meridional wind and (c) relative humidity. Also shown are the potential temperature (dashed grey lines; contour interval is $5 \mathrm{~K}$ with the $315 \mathrm{~K}$ contour solid) and the jet stream winds (bold grey lines; values are 50, 60, 70 and $80 \mathrm{~ms}^{-1}$ ). All panels show the flight track (black line), the sonde release points (arrows) and the locations A and B from Fig. 1. Each sonde profile has been smoothed in the vertical with a $250 \mathrm{~m}$ Gaussian kernel filter before plotting, as described in the text.

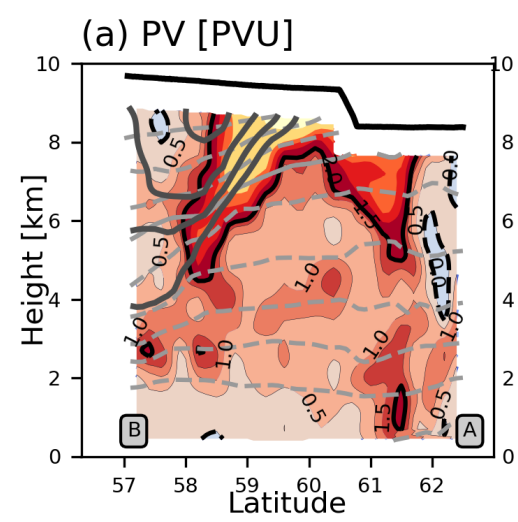

(b) $\left(f-u_{y}\right) \theta_{z} / \rho[\mathrm{PVU}]$

(c) $u_{z} \theta_{y} / \rho[\mathrm{PVU}]$
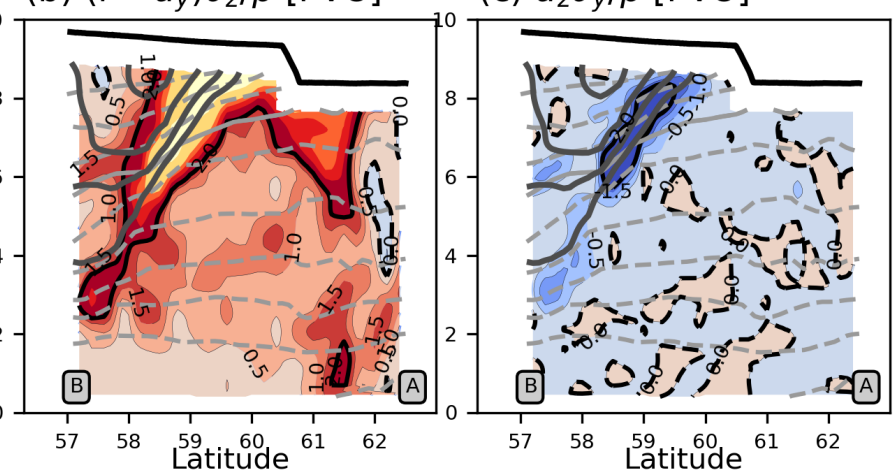

FIGURE 3 PV diagnostics computed from the dropsonde data in Fig. 2: (a) the full PV field, (b) the PV associated with the vertical component of vorticity, and (c) the PV associated with the horizontal component of vorticity. The details of the calculations are described in the text. All panels highlight the zero contour (black lines) and the \pm 2 PVU contours (solid black lines). The potential temperature and jet stream winds are also displayed, as in Fig. 2, for reference. 

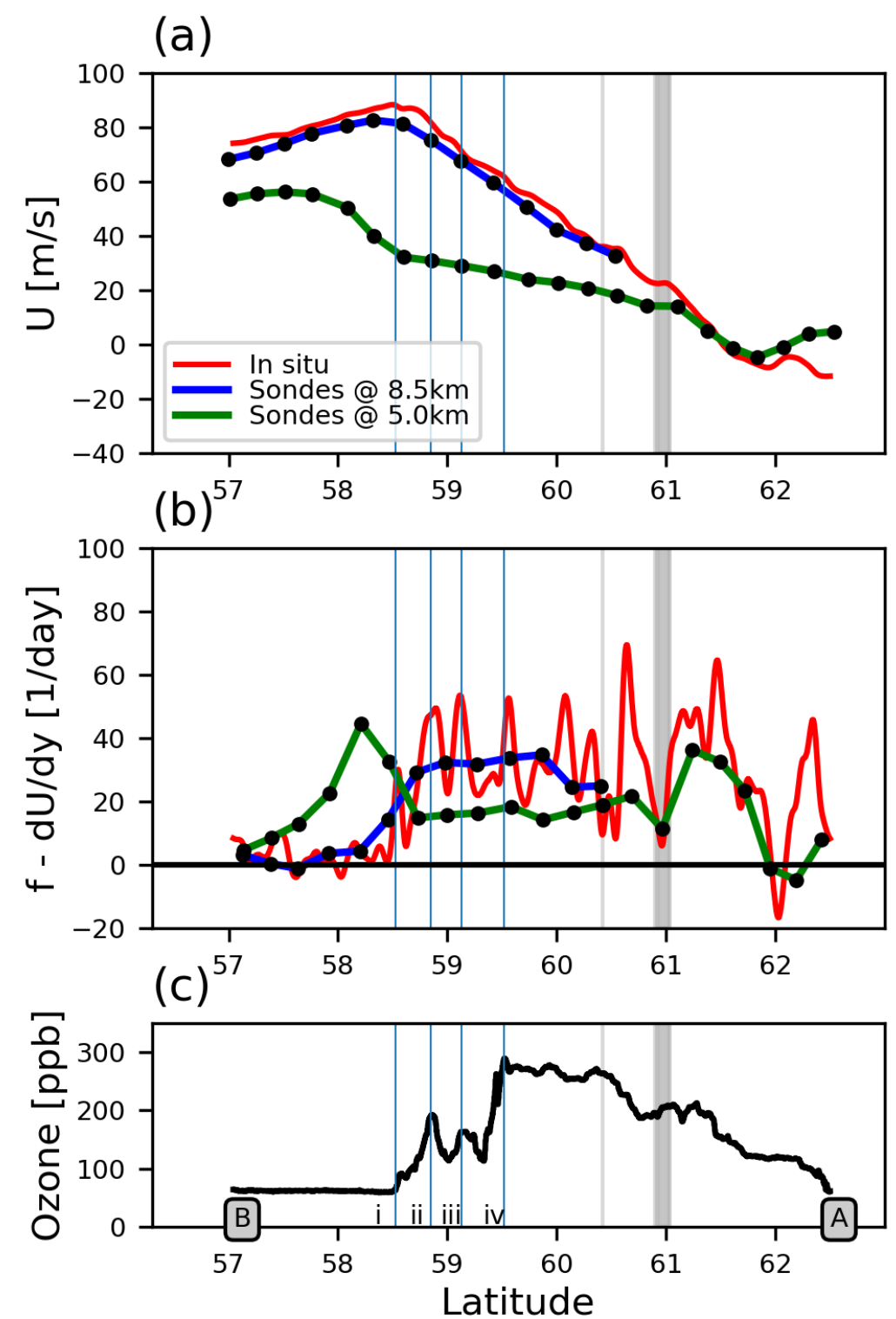

F IG URE 4 In situ observations from FAAM flight B981 on 27 Sep: (a) wind speed, (b) the vertical component of absolute vorticity $\left(f-u_{y}\right)$, and (c) ozone mixing ratio. The red lines in (a) and (b) show in situ wind data, and the blue and green lines show the corresponding values from the dropsonde section in Fig. 2 at $8.5 \mathrm{~km}$ and $5 \mathrm{~km}$ respectively. Regions where the aircraft roll angle is greater 2 degrees, and the wind data is liable to inaccuracies, are masked grey. The vertical markers $\mathrm{i}-\mathrm{iv}$ are referred to in the text. 
(a)

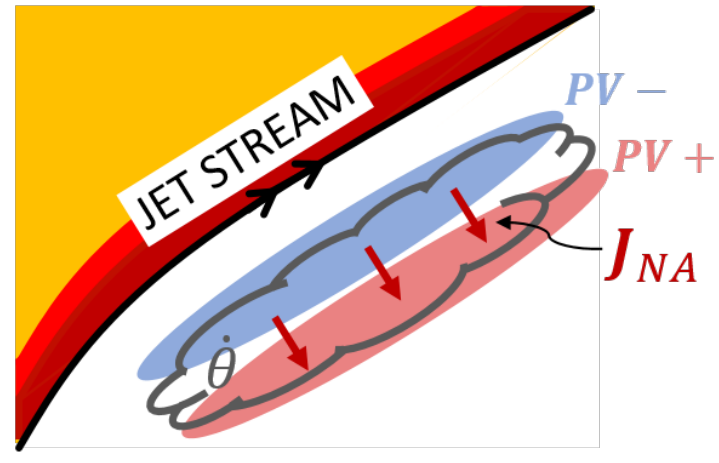

(b)

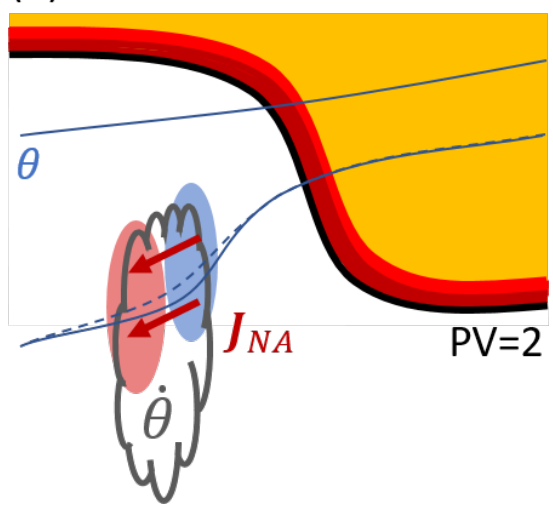

F I GURE 5 A schematic showing the orientation of the non-advective PV flux $\mathrm{J}_{N A}$ in a typical WCB situation. Panel (a) shows a plan view of the jet stream (black line) on an isentropic surface, and heating occurs on its southern flank below the altitude of the jet maximum (grey cloud). From (17), it can be anticipated that the non-advective PV flux will lie across the region of heating and be directed away from the jet core (red arrows). The PV will reduce closest to the jet core (blue shading) and increase further south (red shading). Panel (b) shows a cross section of the same situation, with the tropopause (black line) and potential temperature contours (blue lines; dashed = before heating, solid = after) indicated. The non-advective flux is directed down the isentropic slope 
(a) Initial Wind and StrmFn

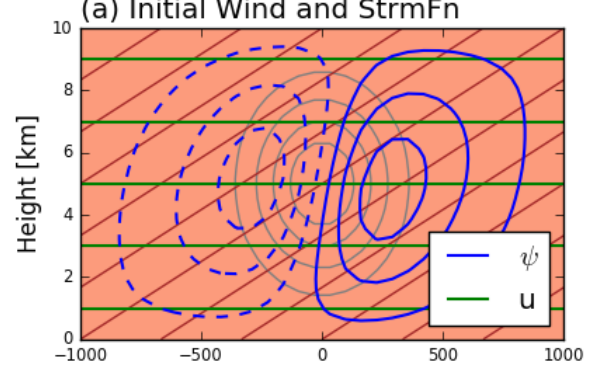

(c) Final Wind

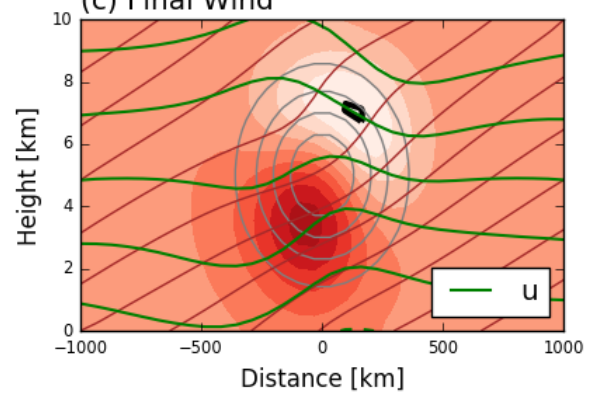

(b) Initial Isentropic PV Terms

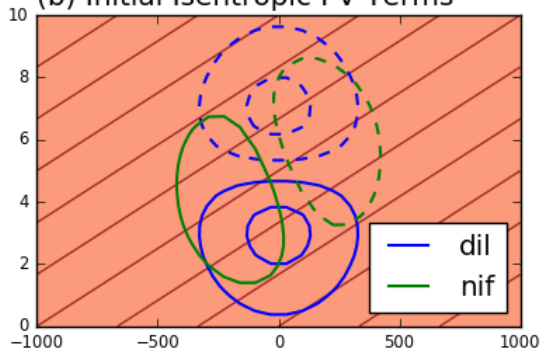

(d) Final Isentropic PV Terms

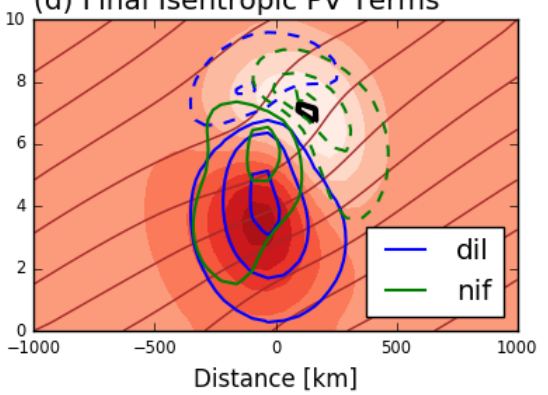

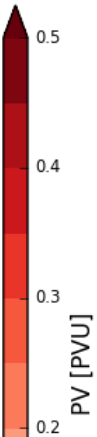

0.1

0.0

F I G URE 6 Case 1 frontal-geostrophic simulation. Panels show PV (shading) and potential temperature (brown contours; interval $5 \mathrm{~K}$ ) at times $t=0$ (panels (a) and (b)) and $t=18 \mathrm{hrs}$ (panels (c) and (d)). Panels (a) and (c) also show the zonal wind (green contours; interval $5 \mathrm{~m} \mathrm{~s}^{-1}$ ), the heating rate (grey contours; interval $2 \mathrm{~K} \mathrm{day}^{-1}$ ) and the meridional streamfunction (blue contours; interval $1000 \mathrm{~kg} \mathrm{~m}^{2} \mathrm{~s}^{-1}$, shown in panel (a) only for clarity). Panels (b) and (d) also show the dilution and non-advective flux terms from the isentropic PV tendency equation (blue and green contours; interval 0.1 PVU day $^{-1}$ ). In panels (c) and (d) the zero PV contour is shown in black. 
(a) Initial Wind and StrmFn

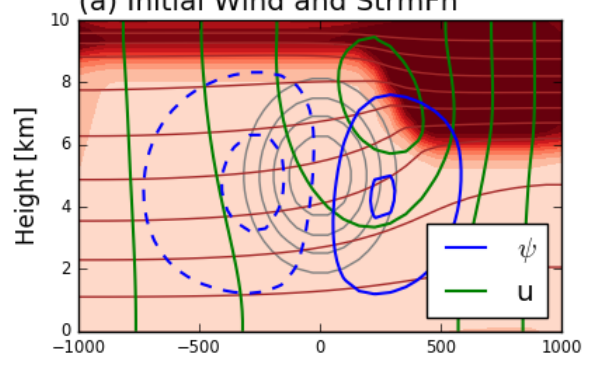

(c) Final Wind

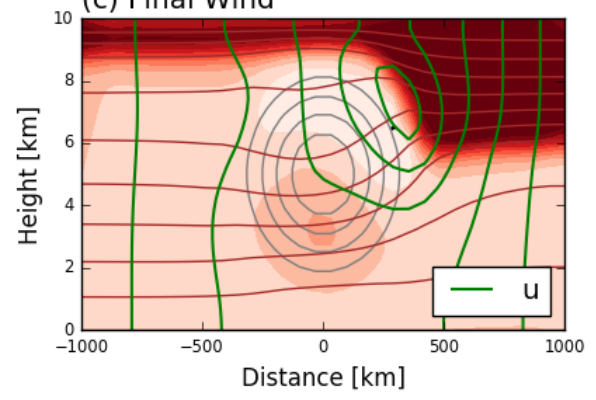

(b) Initial Isentropic PV Terms

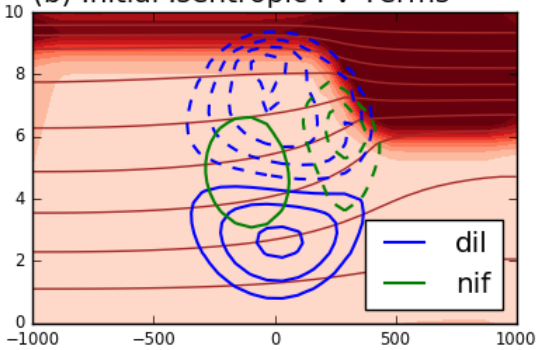

(d) Final Isentropic PV Terms

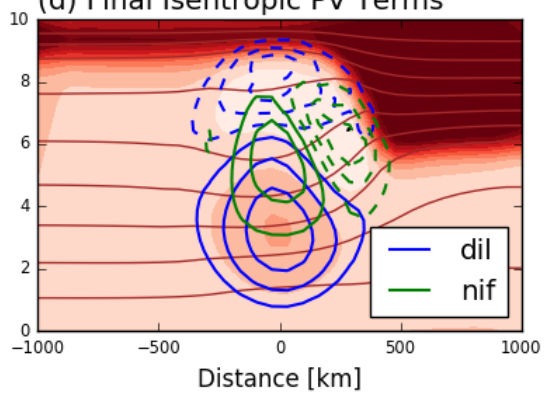

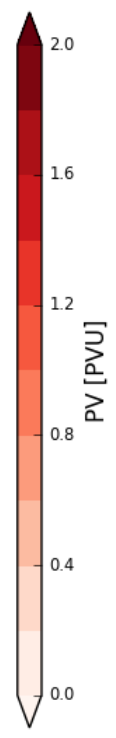

FIGURE 7 Case 2 frontal-geostrophic simulation. Panels show the same variables as Fig. 6. 
(a) Full PV

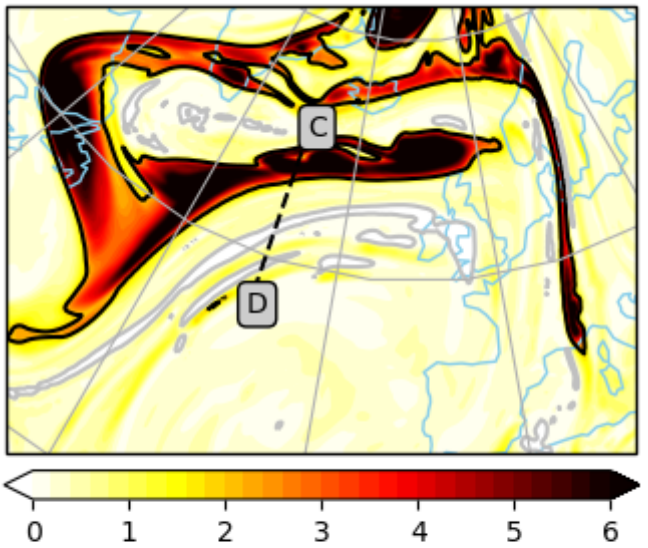

(c) PV change due to all physics

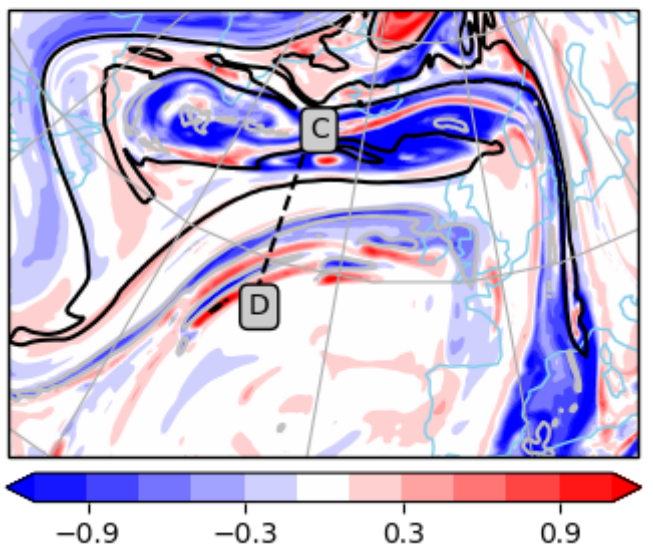

(b) Advected initial PV

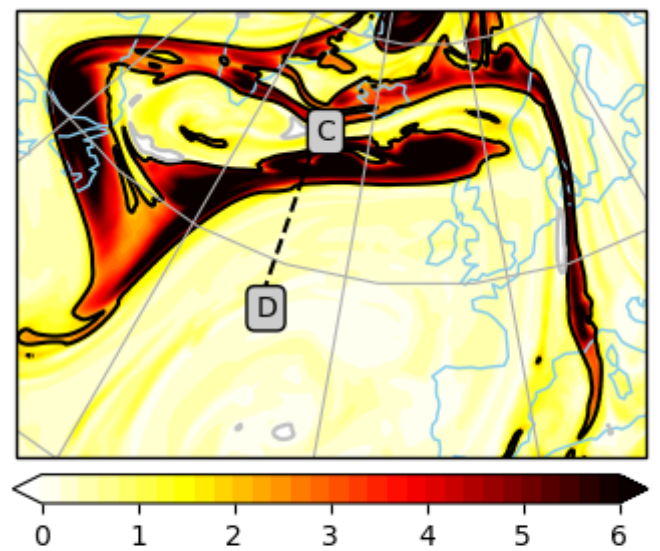

(d) PV change due to dyn. core

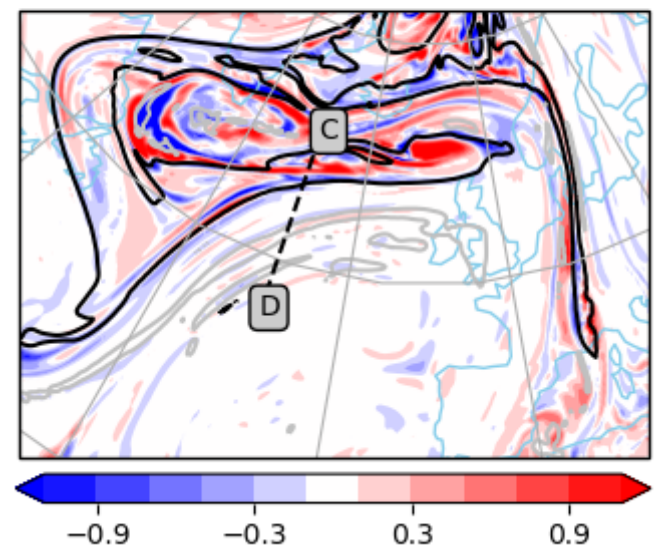

FIGURE 8 PV tracer fields at leadtime T+36 from a MetUM simulation of the ex-tropical storm Karl system. Forecast start time is $00 Z$ on 26 September 2016. Panels show fields at $8.2 \mathrm{~km}$ altitude of: (a) the full PV, (b) the advection-only PV tracer, (c) the total accumulated PV tendency from all the physics schemes and (d) the accumulated PV tendency from the dynamical core. Panels (a) and (b) show additional contour lines at O PVU (grey) and 2 PVU (black) for emphasis. These same contours from the full PV field are reproduced in panels (c) and (d) for reference. All panels show the track of the HALO aircraft along section CD. 
(a) PV change due to radiation

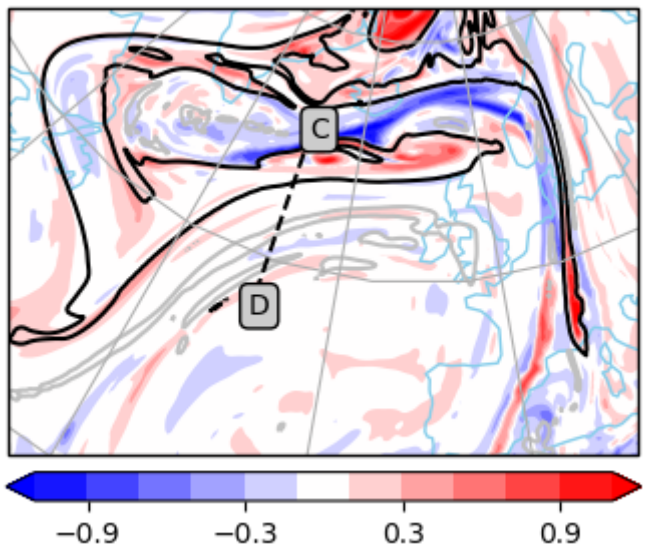

(c) PV change due to turbulent mixing

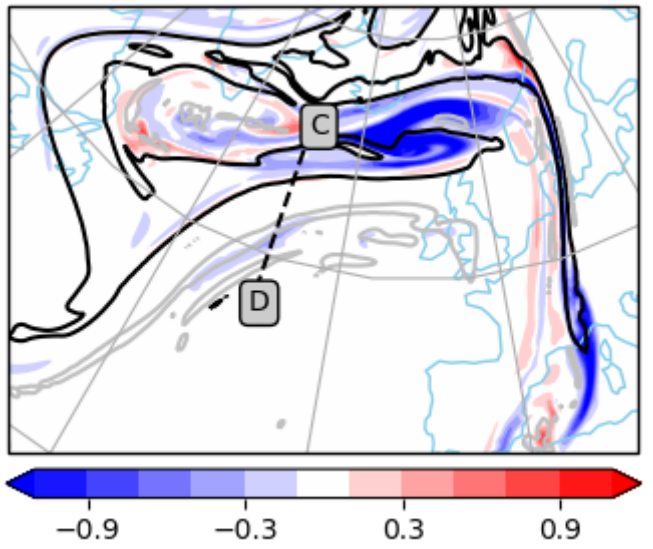

(b) PV change due to large-scale rain

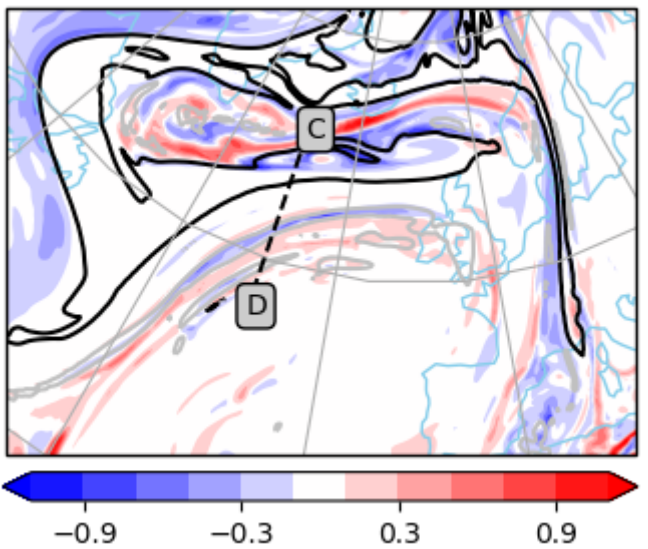

(d) PV change due to convection

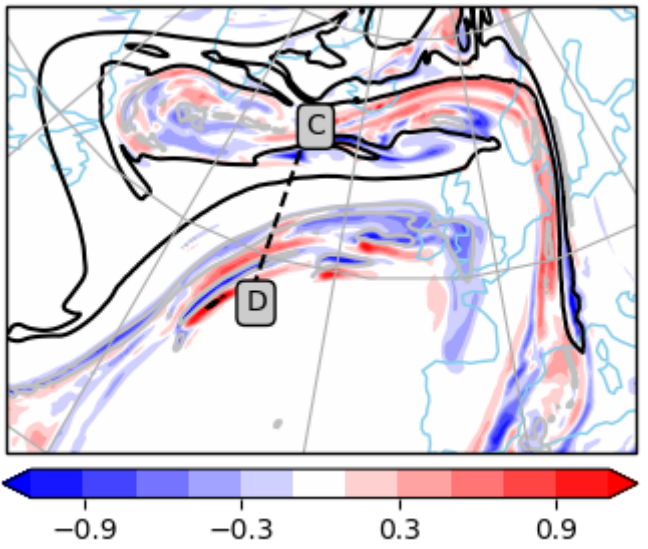

FIGURE 9 As in Fig. 8 except the panels here show the accumulated PV tendency from the four dominant physics schemes separately: (a) radiation, (b) large-scale rain, (c) boundary-layer, and (d) convection. 
(a) Full PV
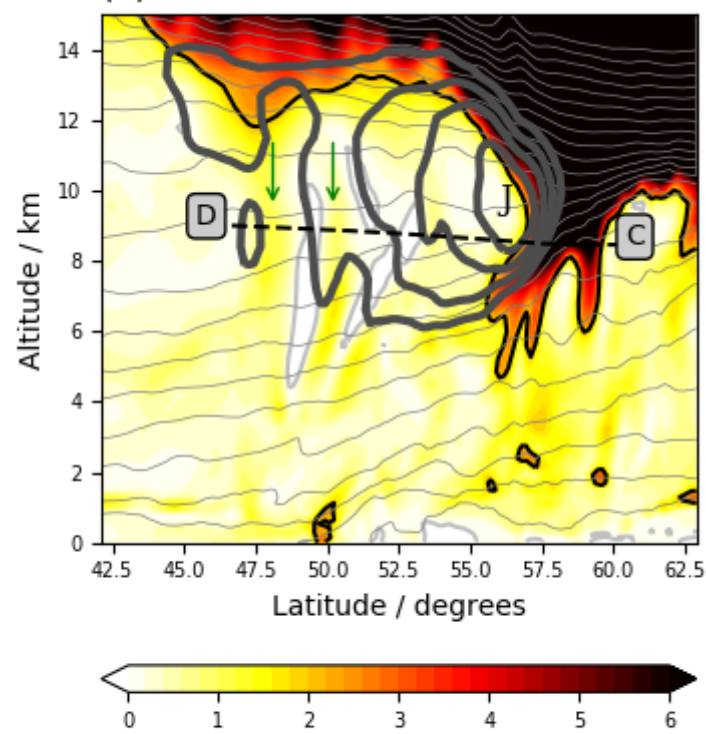

(b) PV change due to all physics
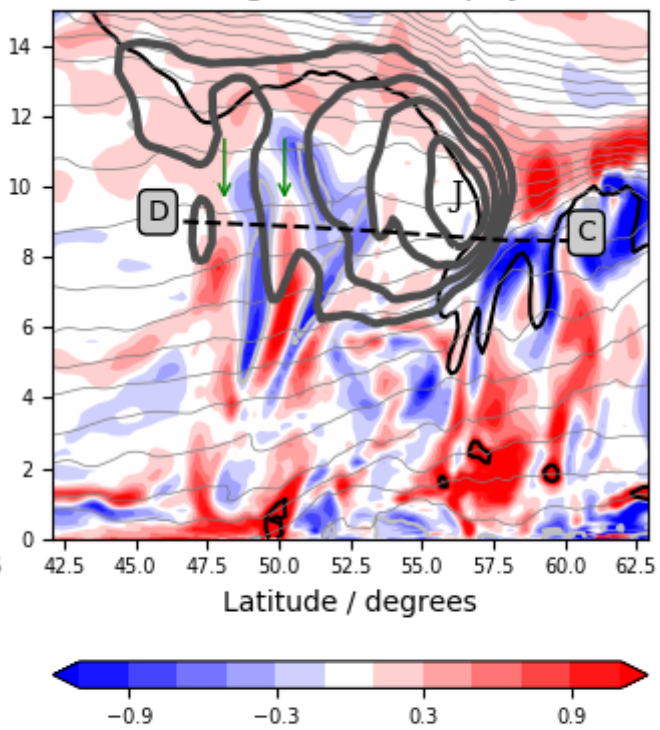

(c) Cross-jet wind shear along HALO flight track

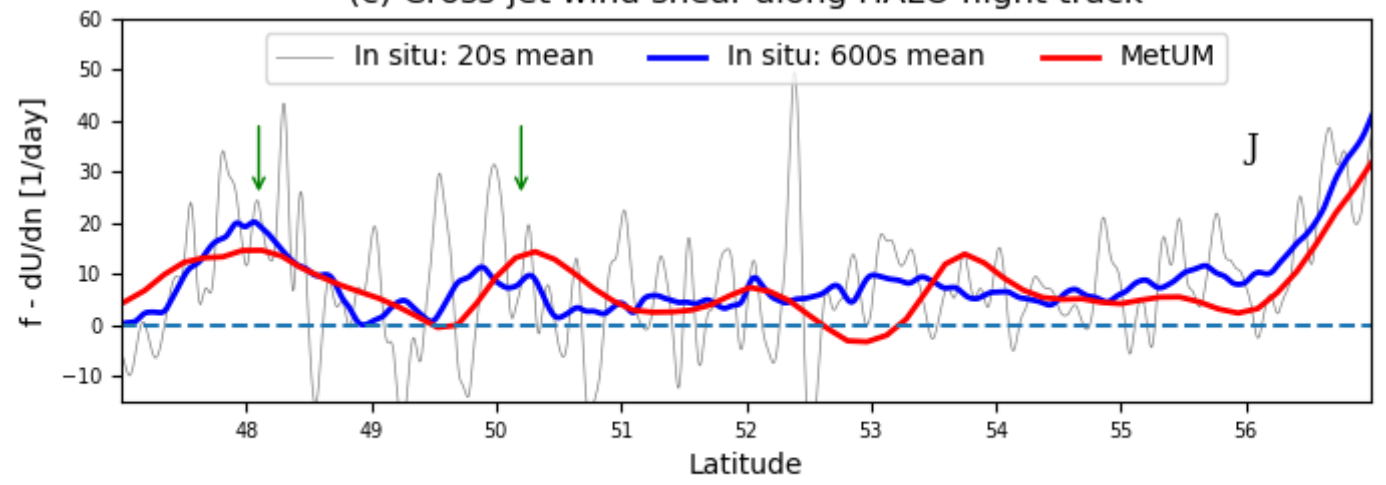

FIGURE 10 Meridional cross sections along 29 W of the MetUM simulation shown in Figs. 8 and 9. Panel (a) shows the full PV and panel (b) shows the total accumulated PV tendency from all the physics schemes. Panel (c) shows the vertical component of absolute vorticity component estimated from in situ wind measurements from the HALO aircraft (grey), the same data with a 10 minute running mean applied (blue), and the same diagnostic computed from the MetUM simulation (red). Panels (a) and (b) also show wind speed contours (bold grey lines; values are 50, 60, 70, and $80 \mathrm{~ms}^{-1}$ ) and the HALO flight section CD. The vertical arrows in all panels indicate positions $48.1 \mathrm{~N}$ and $50.2 \mathrm{~N}$ and the ' $J$ ' indicates the position of the jet maximum. 